\title{
Faults Simulations for Three-Dimensional Reservoir-Geomechanical Models with the Extended Finite Element Method
}

\author{
Jean H. Prevost* \\ Department of Civil and Environmental Engineering \\ Princeton University, Princeton, NJ 08544, USA \\ N. Sukumar \\ Department of Civil and Environmental Engineering \\ University of California at Davis, Davis, CA 95616, USA
}

\begin{abstract}
Faults are geological entities with thicknesses several orders of magnitude smaller than the grid blocks typically used to discretize reservoir and/or over-under-burden geological formations. Introducing faults in a complex reservoir and/or geomechanical mesh therefore poses significant meshing difficulties. In this paper, we consider the strong-coupling of solid displacement and fluid pressure in a three-dimensional poro-mechanical (reservoirgeomechanical) model. We introduce faults in the mesh without meshing them explicitly, by using the extended finite element method (X-FEM) in which the nodes whose basis function support intersects the fault are enriched within the framework of partition of unity. For the geomechanics, the fault is treated as an internal displacement discontinuity that allows slipping to occur using a Mohr-Coulomb type criterion. For the reservoir, the fault is either an internal fluid flow conduit that allows fluid flow in the fault as well as to enter/leave the fault or is a barrier to flow (sealing fault). For internal fluid flow conduits, the continuous fluid pressure approximation admits a discontinuity in its normal derivative across the fault, whereas for an impermeable fault, the pressure approximation is discontinuous across the fault. Equal-order displacement and pressure approximations are used. Two- and three-dimensional benchmark computations are presented to verify the accuracy of the approach, and simulations are presented that reveal the influence of the rate of loading on the activation of faults.
\end{abstract}

KEYWORDS: Finite elements, X-FEM, Poro-mechanics, Reservoir, Geomechanics, Multiphysics coupling, Faults, Discontinuities

\section{HIGHLIGHTS:}

- Fault as a displacement discontinuity (stress) and a pressure discontinuity (sealing fault) or a continuous pressure but discontinuous normal gradient discontinuity (flow conduit)

- X-FEM implementation (in both 2D and 3D)

- Fully coupled poro-mechanical effects (stress and pressure)

- Influence of rate of loading on faults reactivation is demonstrated

*Corresponding Author, email: prevost@princeton.edu 


\section{INTRODUCTION}

Faults are geological fractures of rock in which there has been relative displacement in the plane of fracture. Fault slip reactivation may be triggered by changes in hydraulic pressures and deformations of the rock matrix, which occur during injection and/or extraction of resident fluids as a result of disposal of waste water or $\mathrm{CO}_{2}$, or as a result of hydrocarbon extraction. The motion of faults due to the injection or removal of fluids is known to induce seismicity (National Research Council, 2012), and for potentially generating leaks in containment scenarios. The potential for fault reactivation associated with industrial activities is an important problem not just from a safety viewpoint, but also from a public acceptance perspective. It is becoming increasingly apparent that accurate and reliable simulation techniques are needed that can capture the solid displacement, pressure, and thermal and saturation effects in reservoir-geomechanical models. In this paper, we consider the strong coupling between solid displacement and fluid pressure in a three-dimensional reservoir-geomechanical model, and present extended Finite Element simulations that reveal the influence of loading rates on the activation of faults.

Mechanical and hydraulic characterization of faults have been the subject of many experimental studies (Barton, 2013; Evans et al., 1997; Faulkner et al., 2003; Jaeger and Cook, 1969; Mizoguchi et al., 2008; Wibberley and Shimamoto, 2003). The concept of a Mohr-Coulomb shear strength description in terms of a cohesion and friction angle is widely accepted, albeit with some caveats (Barton, 2013). Hydraulic description in terms of permeability is more controversial. As discussed in Rubin (2015), fault zones most often act as barriers to a cross-fault (impermeable fault) flow, and some that are usually active, act as conduits for along-fault flow. Permeability numbers from the fault zone that hosted the Kobe earthquake in Japan have been reported to be $10^{-20} \mathrm{~m}^{2}$ for the very fine-grained core of the fault zone; and $10^{-16} \mathrm{~m}^{2}$ for the surrounding zone of damaged rock. A nearly impermeable fault core surrounded by a fractured host rock is a prescription for a faultnormal permeability that is much less than that of the host rock, and for an along-fault permeability that is much higher. As reported by Rubin (2015), of all the rock properties that are commonly measured, permeability is among the most wildly varying. Permeability depends on the confining pressure, amount of fault slip, etc. Note that the very low permeability of $10^{-20} \mathrm{~m}^{2}$ applies to regions only millimeters across.

Faults are geological entities with thicknesses several orders of magnitude smaller than the grid blocks typically used to discretize reservoir and/or over-under-burden geological formations. Coates and Schoenberg (1995) used finite-difference to model faults with a displacement discontinuity across it. Since this initial work, finite elements have been adopted for faults modeling. However, introducing faults in a complex reservoir and/or geomechanical finite element mesh presents significant difficulties due to the need to generate very refined meshes in the vicinity of the faults. Several researchers have recently focused on fault modeling in geomaterials (see, e.g. Cappa and Rutqvist, 2011; Rinaldi et al., 2014), but most of the studies are limited to two dimensions and only approximately account for the coupling between fluid flow and solid 
deformation that occurs in fluid-saturated porous media (so-called poro-mechanical effects). Furthermore, these implementations are restricted to sealing faults, and do not fully address the challenge of inserting a fault within a mesh. A recent study (Jha and Juanes, 2014) addresses this challenge in both 2D and 3D by modeling faults as surfaces of discontinuity using interface elements (fault must conform to element boundaries) and Lagrange multipliers, but it is also restricted to sealing faults. Early theoretical work on issues related to embedding strong discontinuities in saturated porous materials can be found in Armero and Callari (1999). In the present paper, we use the extended finite element method (X-FEM) to introduce faults without the need to mesh them explicitly. Numerical results using the X-FEM have been presented in 2D for fractured porous media (de Borst et al., 2006; Fumagalli and Scotti, 2014; Lamb et al., 2013; Réthoré et al., 2007; Talebian et al., 2013), and for 3D hydraulic fracture simulations (Gupta and Duarte, 2014; Secchi and Schrefler, 2012). The implementation of the X-FEM in 3D (Sukumar et al., 2000) is significantly more complex than in 2D (Moës et al., 1999).

In the three-dimensional model for the geomechanics, we treat the fault as an internal displacement discontinuity that allows slipping to occur using a Mohr-Coulomb type criterion. For the reservoir, the fault is either an internal fluid flow conduit that permits fluid flow to occur within the fault as well as to enter or leave the fault, or is a barrier to flow. In the X-FEM, the faults are represented by enriching the displacement approximation with a discontinuous function via the framework of partition-of-unity (Melenk and Babuška, 1996). For sealing/impermeable faults, a pressure discontinuity must occur across the fault, and a discontinuous function is used to model the pressure discontinuity across the fault. For fault as a fluid flow conduit, the transverse permeability is typically several orders of magnitude smaller than the host. Conversely, the longitudinal permeability can be larger than the host. For this case, one must use a continuous pressure function that permits a discontinuous normal pressure gradient across the fault. For internal fluid flow conduits, a $\mathrm{C}^{0}$ continuous function is used for the fluid pressure approximation that admits a discontinuity in its normal derivative across the fault (Moës et al., 2003; Sukumar et al., 2001). For the standard finite element contribution, equal-order solid displacement and pressure approximations are used. As shown and discussed in detail in Prevost (2013), two-way coupling of pressure and stress equations is required if poro-mechanical effects are to be accurately captured. Also, as shown in Prevost (2013), one-way inexpensive iterative (sequential) integration of reservoir-geomechanical equations can work, but requires a very large number of iterations for accurate integration of such strongly-coupled equations. This is not surprising since it is wellknown that fixed-point iterations, if they converge at all, require a large number of iterations to converge.

This paper is organized as follows. Section 2 outlines the poro-mechanical field equations, and the essentials of the weak formulation are described in Section 3. The displacement and pressure approximations in the modeling of faults using the X-FEM are presented in Section 4, with details on the residual contributions from the stress and pressure equations. Central to the success of the 
fully coupled implementation is the computation of the Jacobian matrix, which is discussed in Section 5. The elemental contributions to the coupling Jacobian matrix are computed through numerical finite-differencing of the residuals (Preisig and Prevost, 2011a, 2012 ; Prevost, 1981, 2013; Prevost, 2014). In Section 6, numerical results in 2D and 3D are presented that affirm the versatility and sound accuracy of the method.

\section{FIELD EQUATIONS}

Detailed derivations of the poro-mechanical equations can be found in Coussy (2004). For an isothermal fully saturated porous solid, stress and pressure are governed by the stress momentum equations:

$$
\nabla \cdot \boldsymbol{\sigma}+\rho \mathbf{g}=0 \quad \boldsymbol{\sigma}=\boldsymbol{\sigma}^{\prime s}-b p_{f} \boldsymbol{\delta},
$$

the balance of mass equation:

$$
\varphi \frac{d \rho_{f}}{d t}+\nabla \cdot\left(\rho_{f} \overline{\mathbf{q}}_{f}\right)+\rho_{f} \frac{d \varphi}{d t}=0,
$$

where $\overline{\mathbf{q}}_{f}=-\frac{1}{\mu_{f}} \mathbf{k} \cdot\left[\nabla p_{f}-\rho_{f} \mathbf{g}\right]$ is the Darcy flux, and the porosity equation:

$$
\frac{d \varphi}{d t}=b \nabla \cdot \mathbf{v}^{S}+\frac{1}{N} \frac{d p_{f}}{d t},
$$

where $\frac{1}{N}=\frac{b-\varphi_{0}}{K_{S}}$. For a slightly compressible fluid, the balance of mass and the porosity equation can be combined to yield the pressure equation:

$$
\frac{1}{M} \frac{d p_{f}}{d t}+\nabla \cdot \overline{\mathbf{q}}_{f}+b \nabla \cdot \mathbf{v}^{S}=0
$$

where $\frac{1}{M}=\frac{1}{N}+\frac{\varphi}{K_{f}}$. In (1) - (4), $\boldsymbol{\sigma}$ is the total Cauchy stress, $\boldsymbol{\sigma}^{\prime s}$ is effective stress (Terzaghi, 1923), $b=1-\frac{K^{S}}{K_{S}}$ is the Biot coefficient (Biot, 1941, 1955), $\rho=(1-\varphi) \rho_{S}+\varphi \rho_{f}$ is the total mass density, $\rho_{S}$ and $\rho_{f}$ are the solid and fluid mass densities, respectively, $\varphi$ is the porosity, $\varphi_{0}$ is the initial porosity, $\mathbf{g}$ is the body force, $\mathbf{k}$ is the permeability, $\mu_{f}$ is fluid viscosity, $\overline{\mathbf{q}}_{f}$ is the Darcy flux, and $K_{s}$ and $K_{f}$ are the solid (grains) and fluid bulk moduli, respectively. It is clear that the pressure equation is coupled to the stress equation through the divergence of the solid velocity $\nabla \cdot \mathbf{v}^{S}$. The solid effective stress is related to the solid strains (solid deformation gradient). Assuming, for simplicity, a linear elastic porous skeleton

$$
\boldsymbol{\sigma}^{\prime S}=\left(K^{S}-\frac{2 G^{S}}{3}\right) \varepsilon_{V}^{S} \boldsymbol{\delta}+2 G^{S} \boldsymbol{\varepsilon}^{S} \quad \text { and } \quad \varepsilon_{V}^{S}=\nabla \cdot \mathbf{u}^{S}
$$


where $K^{S}$ and $G^{S}$ are the bulk and shear moduli (drained) of the solid skeleton, respectively, and $\boldsymbol{\varepsilon}^{S}$ is the symmetric part of the solid displacement gradient. It is important to realize that diffusion time is controlled by the diffusion coefficient (see, e.g. Coussy, 2004)

$$
c_{f}=\frac{k}{\mu_{f}} M \frac{\lambda^{S}+2 \mu^{S}}{\lambda^{S}+2 \mu^{S}+b^{2} M},
$$

where $\lambda^{s}+2 \mu^{S}=K^{S}+\frac{4}{3} G^{s}$.

\section{WEAK FORMULATION}

The weak formulation corresponding to the coupled partial differential equations (1) and (4) is obtained by proceeding along standard lines (see, e.g. Hughes, 1987). Both stress and pressure equations are multiplied by appropriate test functions for the displacement and the pressure and then integrated over the volume of the domain $\Omega$. Invoking the divergence theorem to push volume integrals on the boundary of the domain $\partial \Omega-\Gamma_{c}$ and the fault $\Gamma_{c}$, leads to the weak forms.

\subsection{Stress equation}

Let $\mathbf{w}$ be a vector with components $w_{i} \in H_{0}^{1}\left(\Omega-\Gamma_{c}\right)$ with $[\mathbf{w}]=\mathbf{w}^{+}-\mathbf{w}^{-}$representing the jump discontinuity across surface $\Gamma_{c}$. On taking the dot product of (1) by $\mathbf{w}$ and integrating over the volume $\Omega$, we obtain

$$
\int_{\Omega}(\nabla \cdot \boldsymbol{\sigma}) \cdot \mathbf{w} d \Omega+\int_{\Omega} \rho \mathbf{g} \cdot \mathbf{w} d \Omega=0
$$

Then, on integrating by parts we have

$$
\int_{\Omega} \boldsymbol{\sigma}: \nabla \mathbf{w} d \Omega=\int_{\Omega} \nabla \cdot(\boldsymbol{\sigma} \cdot \mathbf{w}) d \Omega+\int_{\Omega} \rho \mathbf{g} \cdot \mathbf{w} d \Omega .
$$

Finally, using the divergence theorem in $\Omega$ and symmetry of $\boldsymbol{\sigma}$ yields

$$
\int_{\Omega} \boldsymbol{\sigma}: \nabla_{0} \mathbf{w} d \Omega=\int_{\partial \Omega_{h}-\Gamma_{c}} \mathbf{h} \cdot \mathbf{W} d \Gamma-\int_{\Gamma_{c}}(\boldsymbol{\sigma} \cdot \mathbf{n}) \cdot[\mathbf{w}] d \Gamma+\int_{\Omega} \rho \mathbf{g} \cdot \mathbf{w} d \Omega,
$$

where $\mathbf{h}=\boldsymbol{\sigma} \cdot \mathbf{n}$ is the prescribed traction on $\partial \Omega_{h}$ and $\nabla_{0} \mathbf{w}$ is the symmetric part of the gradient of $\mathbf{w}$. The boundary, $\partial \Omega$, is defined such that $\partial \Omega=\partial \Omega_{\mathbf{u}} \cup \partial \Omega_{h}, \partial \Omega_{\mathbf{u}} \cap \partial \Omega_{h}=\varnothing$.

\subsection{Pressure equation}

We consider two cases, a sealing fault and a fault as a flow conduit. For the sealing fault, let $v_{f} \in H_{0}^{1}\left(\Omega-\Gamma_{c}\right)$ be a scalar with $\left[v_{f}\right]=v_{f}^{+}-v_{f}^{-}$representing the jump discontinuity across the surface $\Gamma_{c}$. For the flow conduit, let $v_{f} \in H_{0}^{1}(\Omega)$ be a scalar with $\left[\mathbf{n} \bullet \nabla v_{f}\right]$ representing the normal gradient jump discontinuity across the surface $\Gamma_{c}$. Multiplying (4) by $v_{f}$ and integrating over the volume $\Omega$ yields 


$$
\int_{\Omega}\left[\frac{1}{M} \frac{d p_{f}}{d t}+\nabla \cdot \overline{\mathbf{q}}_{f}+b \nabla \cdot \mathbf{v}^{s}\right] v_{f} d \Omega=0
$$

where $\overline{\mathbf{q}}_{f}=-\frac{1}{\mu_{f}} \mathbf{k} \cdot\left[\nabla p_{f}-\rho_{f} \mathbf{g}\right]$. Then, integration by parts yields

$$
\int_{\Omega} \overline{\mathbf{q}}_{f} \cdot \nabla v_{f} d \Omega=\int_{\Omega} \nabla \cdot\left(\overline{\mathbf{q}}_{f} v_{f}\right) d \Omega+\int_{\Omega}\left[\frac{1}{M} \frac{d p_{f}}{d t}+b \nabla \cdot \mathbf{v}^{S}\right] v_{f} d \Omega .
$$

For the sealing fault, using the divergence theorem in $\Omega$ results in

$$
\int_{\Omega} \overline{\mathbf{q}}_{f} \cdot \nabla v_{f} d \Omega=\int_{\partial \Omega_{q_{f}}-\Gamma_{c}} h_{q_{f}} v_{f} d \Gamma-\int_{\Gamma_{c}}\left(\overline{\mathbf{q}}_{f} \cdot \mathbf{n}\right)\left[v_{f}\right] d \Gamma+\int_{\Omega}\left[\frac{1}{M} \frac{d p_{f}}{d t}+b \nabla \cdot \mathbf{v}^{S}\right] v_{f} d \Omega .
$$

For the flow conduit, using the divergence theorem in $\Omega$ results in

$$
\int_{\Omega} \overline{\mathbf{q}}_{f} \cdot \nabla v_{f} d \Omega=\int_{\partial \Omega_{q_{f}}-\Gamma_{c}} h_{q_{f}} v_{f} d \Gamma-\int_{\Gamma_{c}}\left[\overline{\mathbf{q}}_{f} \cdot \mathbf{n}\right] v_{f} d \Gamma+\int_{\Omega}\left[\frac{1}{M} \frac{d p_{f}}{d t}+b \nabla \cdot \mathbf{v}^{S}\right] v_{f} d \Omega .
$$

In (12) and (13), $h_{q_{f}}=\overline{\mathbf{q}}_{f} \cdot \mathbf{n}$ is the prescribed fluid flux on $\partial \Omega_{q_{f}}$ and the boundary, $\partial \Omega$, is defined such that $\partial \Omega=\partial \Omega_{p_{f}} \cup \partial \Omega_{q_{f}}, \partial \Omega_{p_{f}} \cap \partial \Omega_{q_{f}}=\varnothing$. It is important to note that the contribution of the fault results in a surface integral in both stress and pressure equations.

\section{FAULTS MODELING WITH THE X-FEM}

The discretized finite element equations are obtained by using finite element basis functions that are augmented by X-FEM functions to represent the fault $\Gamma_{c}$. Let $N^{i}(\mathbf{x})$ denote the standard finite element shape functions for a single finite element, where $i \in \mathbb{N}$, the set consisting of nodes that belong to the element. Let $\mathbb{N}_{\text {cut }}$ denote the set of nodes whose basis function support is cut by the fault $\Gamma_{c}$ (see Fig. 1). Then the following approximations are used:

- a discontinuous displacement field (stress equation) (Moës et al., 1999):

$$
\mathbf{u}=\sum_{i \in \mathbb{N}} N^{i}(\mathbf{x}) \mathbf{u}^{i}+\sum_{i \in \mathbb{N}_{c u t}} N^{i}(\mathbf{x}) H_{\Gamma_{c}}(\mathbf{x}) \hat{\mathbf{u}}^{i},
$$

where $H_{\Gamma_{c}}(\mathbf{x})$ is the discontinuous generalized Heaviside function;

- a continuous pressure field (with fluid as a flow conduit):

$$
p_{f}=\sum_{i \in \mathbb{N}} N^{i}(\mathbf{x}) p_{f}^{i}+\sum_{i \in \mathbb{N}_{\text {cut }}} N^{i}(\mathbf{x}) \psi(\mathbf{x}) \hat{p}_{f}^{i},
$$

where $\psi(\mathbf{x})$ is the distance function with a discontinuous normal derivative across $\Gamma_{c}$;

- $\quad$ and a discontinuous pressure field (with a sealing fault):

$$
p_{f}=\sum_{i \in \mathbb{N}} N^{i}(\mathbf{x}) p_{f}^{i}+\sum_{i \in \mathbb{N}_{\text {cut }}} N^{i}(\mathbf{x}) H_{\Gamma_{c}}(\mathbf{x}) \hat{p}_{f}^{i} .
$$

For the stress equation each node $i$ is assigned nsd (number of spatial dimensions) displacement degrees of freedom $\mathbf{u}^{i}$. The set of nodes $\mathbb{N}_{c u t}$ whose basis function support is cut by the fault are 
assigned additional $n s d$ degrees of freedom $\hat{\mathbf{u}}^{i}$. Similarly for the pressure equation, each node $i$ is assigned one pressure degree of freedom $p_{f}^{i}$ and the nodes in $\mathbb{N}_{c u t}$ are assigned an additional pressure degree of freedom $\hat{p}_{f}^{i}$. The resulting semi-discrete finite element equations are integrated in time by using a first order finite difference time-stepping integrator (typically, backward Euler) for both stress and pressure equations.

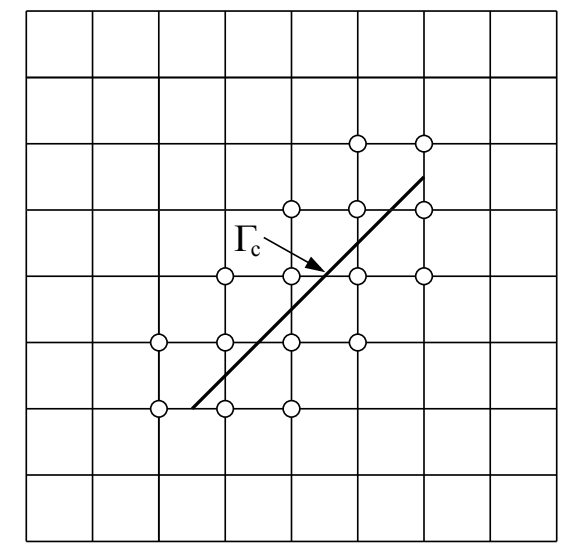

Fig. 1: Enriched nodes (shown as open circles) whose basis function support is cut by the fault $\Gamma_{c}$.

The contribution to the residuals of the elements that are cut by the fault require element partitioning above and below $\Gamma_{c}$ for spatial integration. In 2D, we use triangles as implemented in Sukumar and Prevost (2003). In 3D, we subdivide the cells by using tetrahedral elements (Sukumar et al., 2000). The X-FEM surface integral contributions to the residual equations are computed as follows for both the stress and pressure equations.

\subsection{Residual contribution to stress arising from the surface integral}

From the weak form in (9), the surface integral contribution on $\Gamma_{c}$ is due to $\int_{\Gamma_{c}}(\boldsymbol{\sigma} \cdot \mathbf{n}) \cdot[\mathbf{w}] d \Gamma$. In the $2 \mathrm{D}$ case, the residual is:

$$
\hat{\mathbf{r}}_{\hat{u}}^{a}=-\int_{\Gamma_{c}} 2\left[\hat{\mathbf{B}}^{a}\right]^{T}\left\{\begin{array}{l}
t_{t} \\
t_{n}
\end{array}\right\} d \Gamma,
$$

where $\hat{\mathbf{B}}^{a}=N^{a}\left[\mathbf{e}_{t}, \mathbf{e}_{n}\right]^{T}$ and $t_{t}$ and $t_{n}$ denote the tangential and normal tractions on the plane of the fault, respectively. This is illustrated in Fig. 2 for the two-dimensional (2D) case. Let

$$
[\mathbf{u}]=\mathbf{u}^{+}-\mathbf{u}^{-}
$$

denote the displacement jump across the fault where $\left[u_{n}\right]=[\mathbf{u}] \cdot \mathbf{e}_{n}$ and $\left[u_{t}\right]=[\mathbf{u}] \cdot \mathbf{e}_{t}$. Then, the normal and tangential tractions on the fault are computed as:

$$
t_{n}^{\prime s}=k_{n}\left[u_{n}\right]+p_{f},
$$


where $t_{t}=k_{t}\left[u_{t}\right]$, and $k_{n}$ and $k_{t}$ are penalty coefficients. Note the fluid pressure contribution to the normal total traction results in the effective normal traction $t_{n}^{\prime s}$. The Mohr-Coulomb unilateral constraint requires that if $\left[u_{n}\right]<0$,

$$
\left|t_{t}\right| \leq c_{u}+\left|t_{n}^{\prime}\right| \tan \varphi_{u}
$$

where $c_{u}$ and $\varphi_{u}$ denote cohesion and internal friction angle assigned to the fault. The MohrCoulomb constraint is enforced by using the return correction procedure shown in Fig. 2b. In the three-dimensional case, the fault is a plane. The unit vectors tangent to the surface $\Gamma_{c}, \mathbf{e}_{t}^{1}$ and $\mathbf{e}_{t}^{2}$ are constructed as in laminar shells (see, e.g. Hughes, 1987) and $\mathbf{e}_{n}=\mathbf{e}_{t}^{1} \times \mathbf{e}_{t}^{2}$.

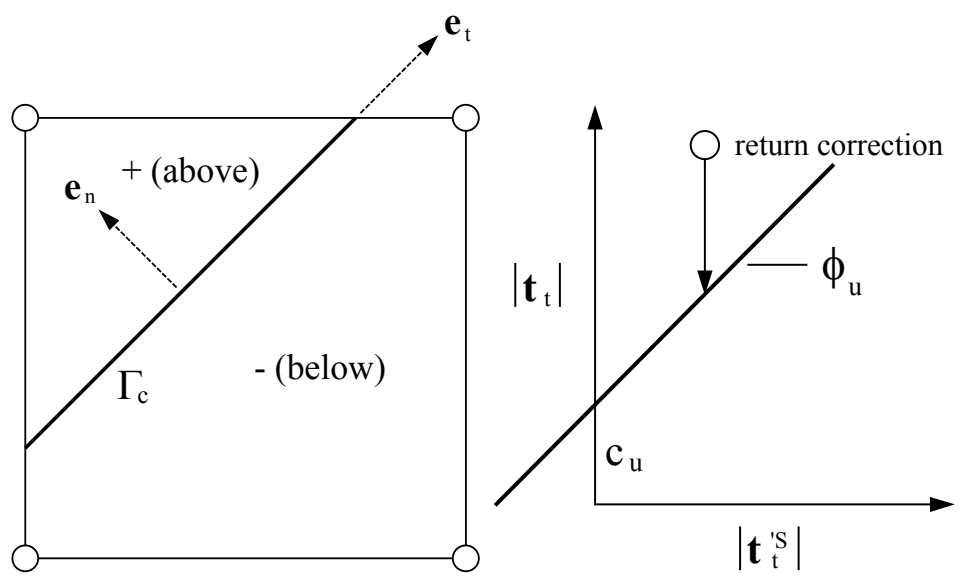

Fig. 2: Fault as a displacement discontinuity (a) geometry, and (b) Mohr-Coulomb constraint.

Other more elaborate models can be used such as slip-weakening models where the mobilized friction angle can be made function of a critical slip $u_{c}$ as

$$
\tan \varphi_{u}=\left\{\begin{array}{cl}
\tan \varphi_{s}-\left(\tan \varphi_{s}-\tan \varphi_{d}\right) \frac{\left|\left[\mathbf{u}_{t}\right]\right|}{u_{c}} & \left|\left[\mathbf{u}_{t}\right]\right| \leq u_{c} \\
\tan \varphi_{d} & \left|\left[\mathbf{u}_{t}\right]\right|>u_{c}
\end{array} .\right.
$$

\subsection{Residual contribution to pressure arising from surface integral}

In the sealing fault case, the surface integral contribution is due to $\int_{\Gamma_{c}}\left(\overline{\mathbf{q}}_{f} \bullet \mathbf{n}\right)[v] d \Gamma$. In the flow conduit case, the surface integral contribution is due to $\int_{\Gamma_{\mathrm{c}}}\left[\overline{\mathbf{q}}_{f} \bullet \mathbf{n}\right] v d \Gamma$. The residual is

$$
\hat{r}_{p}^{a}=-\int_{\Gamma_{c}}\left[\hat{\hat{\mathbf{B}}}^{a}\right]^{T} \cdot \overline{\mathbf{q}}_{f} d \Gamma
$$

where $\hat{\mathbf{B}}^{a}=\left[\hat{\mathbf{B}}^{a}\right]^{+}-\left[\hat{\mathbf{B}}^{a}\right]^{-}$and

$$
\hat{\mathbf{B}}^{a}=\nabla N^{a} \hat{H}_{\Gamma_{c}}^{a}+N^{a} \nabla \hat{H}_{\Gamma_{c}}^{a} .
$$


In the sealing fault case, $\hat{H}_{\Gamma_{\mathrm{c}}}=H_{\Gamma_{\mathrm{c}}}$ represents the discontinuous generalized Heaviside function with $\left[H_{\Gamma_{c}}\right]=H_{\Gamma_{c}}^{+}-H_{\Gamma_{c}}^{-}=2$ on $\Gamma_{c}$. In the fault as a flow conduit, $\hat{H}_{\Gamma_{c}}=\psi_{\Gamma_{c}}$ represents the distance function such that $\psi_{\Gamma_{c}}=0$ on $\Gamma_{c}, \mathbf{n}_{\Gamma_{c}} \cdot \nabla \psi_{\Gamma_{c}}=H_{\Gamma_{c}}$ on $\Gamma_{c}$, and $\left[\mathbf{n}_{\Gamma_{c}} \cdot \nabla \psi_{\Gamma_{c}}\right]=2$ on $\Gamma_{c}$.

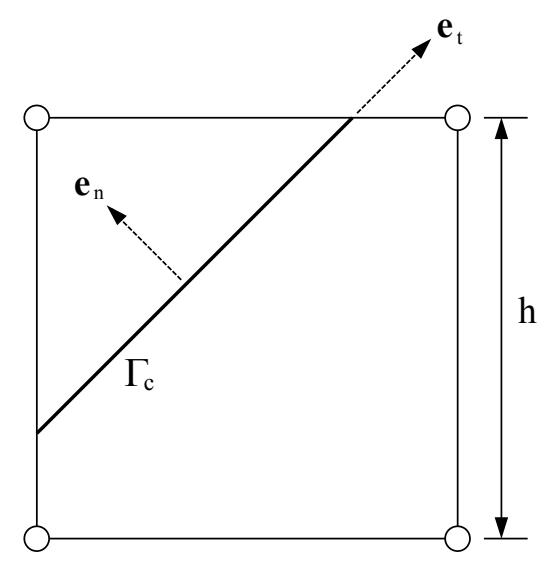

Fig. 3: Fault as a pressure discontinuity or a flow conduit.

The fault thickness $t \approx 10^{-2} h$ where $h$ is the nodal spacing.

The fault is assigned both a longitudinal and a transverse permeability, $k_{t}$ and $k_{n}$ respectively. For a sealing fault $k_{t}=k_{n}$ is a penalty coefficient used to enforce the no flow condition across the fault. For the fault as a flow conduit, the fault is also assigned a thickness $t$. The fault thickness is controlled by the geology, and typically can range from $\mathrm{cm}$ to $\mathrm{mm}$, and therefore $\mathrm{t} \sim 10^{\wedge}-2 \mathrm{~h}$ to $10^{\wedge}-3 \mathrm{~h}$, where $\mathrm{h}$ is the nodal spacing used in a typical reservoir mesh. The finite fault thickness reflects the fact that fluid flow enters and/or leaves both sides of the fault channel. Then in the local axes of the fault, $\mathbf{e}_{t}$ and $\mathbf{e}_{n}$, Darcy's law is assumed to hold as

$$
\left\{\begin{array}{c}
\bar{q}_{t} \\
\bar{q}_{n}
\end{array}\right\}=-\frac{\mathbf{k}_{\text {local }}}{\mu_{f}}\left\{\begin{array}{l}
\partial_{t} p_{f} \\
\partial_{n} p_{f}
\end{array}\right\},
$$

where $\bar{q}_{t}$ and $\bar{q}_{n}$ represent the flow in the fault and

$$
\mathbf{k}_{\text {local }}=\left[\begin{array}{cc}
k_{t} & 0 \\
0 & k_{n}
\end{array}\right] .
$$

Then the flow in the global axes is obtained by performing the rotation between the local and global axes:

$$
\mathbf{k}=\mathbf{R}^{T} \cdot \mathbf{k}_{\text {local }} \cdot \mathbf{R},
$$

where $\mathbf{R}=\left[\mathbf{e}_{t}, \mathbf{e}_{n}\right]^{T}$ and $\overline{\mathbf{q}}_{f}=-\frac{\mathbf{k}}{\mu_{f}} \nabla p_{f}$.

\section{RESIDUAL FORMULATION FOR DISCRETE FINITE ELEMENT EQUATIONS}

Simultaneous integration of the stress and pressure equations requires that a residual formulation be used. The elemental contribution to the Jacobian matrix can then be computed through 
numerical finite differencing of the residuals (see, e.g. Preisig and Prevost, 2011b; Preisig and Prevost, 2012 ; Prevost, 1981) as further explained and detailed hereafter. We use a first-order finite difference time-stepping integrator (typically, backward Euler) for both geomechanical and reservoir equations. Time derivatives are indicated by a superimposed dot $(\bullet)$. The most important elemental residual formulations are summarized hereafter $\left(\Omega^{(e)}\right.$ is the control volume).

For simplicity in notation we omit the contribution to the residuals from the surface integrals in equations (17) and (22).

\subsection{Galerkin stress equation residual formulation}

From (9), the residual in the Galerkin stress equation is:

$$
\mathbf{r}_{u, n+1}^{a}=\mathbf{f}_{u, n+1}^{e x t}-\int_{\Omega^{e}}\left[\mathbf{B}^{a}\right]^{T} \cdot \boldsymbol{\sigma}_{n+1}^{S} d \Omega+\int_{\Omega^{e}} b\left[\nabla N^{a}\right]^{T} p_{n+1}^{(e)} d \Omega,
$$

where the solid displacements are interpolated with the usual shape functions,

$$
\mathbf{u}^{(e)}=\sum_{a=1}^{n e n} N^{a} \mathbf{u}^{a} .
$$

In (26) and (27), nen is the number of nodes per element (e.g., nen $=4$ for a quadrilateral element), and $\mathbf{B}^{a}$ is the usual strain-displacement matrix (see, e.g. Hughes, 1987). The pressure in the element is obtained from the reservoir simulator using appropriate interpolation schemes depending on the specific discretization adopted for the pressure equation.

\subsection{Galerkin pressure equation residual formulation}

From (12) and (13), the residual in the Galerkin pressure equation is:

$$
r_{n+1}^{a}=f_{n+1}^{a, e x t}-\int_{\Omega^{e}} \frac{1}{M} N^{a} \dot{p}_{n+1}^{(e)} d \Omega-\int_{\Omega^{e}}\left[\nabla N^{a}\right]^{T} \cdot \frac{\mathbf{k}}{\mu_{f}} \cdot \nabla p_{n+1}^{(e)} d \Omega-\int_{\Omega^{e}} b N^{a} \nabla \cdot \dot{\mathbf{u}}_{n+1}^{(e)} d \Omega,
$$

where we typically use equal order interpolants for both pressure and solid displacements (see, e.g. Preisig and Prevost, 2011b), i.e.,

$$
p^{(e)}=\sum_{a=1}^{n e n} N^{a} p^{a}
$$

\subsection{Numerical implementation}

Simultaneous integration of the stress and pressure equations is achieved by computing the coupled Jacobian matrix, which is computed by numerical finite differencing of the residuals (see, e.g. Preisig and Prevost, 2011b, 2012 ; Prevost, 1981). To this end, it is convenient to view the global solution vectors and residuals as consisting of contributions from both the geomechanical and reservoir simulator as

$$
\mathbf{x}^{(i)}=\left\{\begin{array}{c}
\mathbf{u}^{(i)} \\
p^{(i)}
\end{array}\right\} \quad \dot{\mathbf{x}}^{(i)}=\left\{\begin{array}{c}
\dot{\mathbf{u}}^{(i)} \\
\dot{p}^{(i)}
\end{array}\right\} \quad \mathbf{r}^{(i)}=\left\{\begin{array}{c}
\mathbf{r}_{u}^{(i)} \\
r_{p}^{(i)}
\end{array}\right\},
$$


where $\mathbf{x}^{(i)}$ is the solution vector at iteration $i$ and lists both nodal displacements and pressures. Then, time-integration is achieved by using the usual generalized trapezoidal predictor-corrector integrator scheme, i.e.,

$$
\mathbf{x}_{n+1}^{(i+1)}=\tilde{\mathbf{x}}_{n+1}+\alpha \Delta t \dot{\mathbf{x}}_{n+1}^{(i+1)} \quad \tilde{\mathbf{x}}_{n+1}=\mathbf{x}_{n}+(1-\alpha) \Delta t \dot{\mathbf{x}}_{n},
$$

where $\alpha=1$ for backward Euler, $\alpha=1 / 2$ for mid-point, and $\Delta t$ is the time step. Then the solution to the nonlinear set of coupled equations is obtained at every iteration as

$$
\dot{\mathbf{x}}_{n+1}^{(i+1)}=\dot{\mathbf{x}}_{n+1}^{(i)}+\delta \mathbf{x}^{(i)} \quad-[\mathbf{J}]^{(i)} \delta \mathbf{x}^{(i)}=\mathbf{r}_{n+1}^{(i)},
$$

where $[\mathbf{J}]^{(i)}$ is the Jacobian determinant matrix obtained by numerical finite-differencing of the residual, i.e.,

$$
\left[J_{P Q}\right]^{(i)}=\frac{\partial r_{P}^{(i)}}{\partial \dot{x}_{Q}^{(i)}} \approx \frac{r_{P}^{(i)}\left(\dot{x}_{Q}^{(i)}+\bar{h}\right)-r_{P}^{(i)}\left(\dot{x}_{Q}^{(i)}\right)}{\bar{h}},
$$

and $P$ and $Q$ are global equation numbers corresponding to unknown displacements and pressures. The Jacobian determinant matrix is computed element-by-element (ebe) through assembly operations, i.e. the ebe Jacobian assembly is

$$
\left[J_{P Q}\right]^{(i)}=A_{e=1, n e l}\left[J_{p q}^{(e)}\right]^{(i)} \text {. }
$$

The local equation numbers are $q$ and $p=i+n d f(a-1)$ with $i=1$, ned and $a=1$, nen. The total number of degrees of freedom at each node is $n d f=n s d+1$. In addition, ned is the local number of degrees of freedom. The global equation numbers are $P=i d(i, i e n(a, e))$ with $A=i e n(a, e)$ being the connectivity. For the geomechanical elements, ned $=n s d$ and $i=1$, nsd, whereas for the reservoir elements, ned $=1$ and $i=n s d+1$. The procedure is detailed in Table 1 . The perturbation of the degrees of freedom $\bar{h}$ is a small parameter chosen as

$$
\bar{h}=\frac{\sqrt[3]{\varepsilon_{M}}}{\mid \dot{x}_{n+1}^{(i)}(\text { idof }, \text { node }) \mid}, \quad \bar{h}=\max \left(\left(\varepsilon_{M}\right)^{1 / 3}, \bar{h}\right),
$$

where $\varepsilon_{M}$ is machine precision. The above procedure is illustrated in Table 1 for a forward evaluation of the Jacobian matrix. It is easily extended to a backward scheme by changing $\bar{h}$ to $-\bar{h}$ and/or to a central difference approximation by using

$$
\left[J_{P Q}\right]^{(i)}=\frac{\partial r_{P}^{(i)}}{\partial \dot{x}_{Q}^{(i)}} \approx \frac{r_{P}^{(i)}\left(\dot{x}_{Q}^{(i)}+\bar{h}\right)-r_{P}^{(i)}\left(\dot{x}_{Q}^{(i)}-\bar{h}\right)}{2 \bar{h}} .
$$

With this modification, the procedure is then twice as expensive and this is usually not warranted. It is important to note that the coupling matrix is never explicitly assembled, rather it is computed by the element-by-element contributions to the residual. 
Table 1: Pseudocode for element-by-element (ebe) Jacobian matrix computation.

//using forward finite-differencing as an example

$[\mathbf{J}] \leftarrow \mathbf{0}$

$n d f \leftarrow n s d+1$

for $e=1$, nel $\quad / /$ nel $=$ number of elements

Compute element contribution to residual, $r_{p, n+1}^{(i)}\left(\dot{\sim}_{n+1}^{(i)}\right), p=1$, nen $\times n d f, i=1, n s d$

for $a=1$, nen

node $\leftarrow$ ien $(a, e) \quad / /$ ien $=$ global node number

for idof $=1, n d f$

$\dot{x}_{n+1}^{(i)}($ idof, node $) \leftarrow \dot{x}_{n+1}^{(i)}($ idof, node $)+\bar{h}$

$\mathbf{x}_{n+1}^{(i)} \leftarrow \tilde{\mathbf{x}}_{n+1}+\alpha \Delta t \dot{\mathbf{x}}_{n+1}^{(i)}$

$\mathbf{u}_{n+1}^{(e)} \leftarrow \sum_{a} N^{a} \mathbf{u}_{n+1}^{a(i)}$

$p_{n+1}^{(e)} \leftarrow \sum_{a} N^{a} p_{n+1}^{a(i)}$

$q \leftarrow i d o f+n d f(a-1)$

if $(e=$ solid element $)$ then

$$
\begin{aligned}
& r_{p, n+1}^{(i)} \leftarrow f_{i, n+1}^{b, e x t}-\mathbf{e}_{i}^{T} \int_{\Omega^{e}}\left[\mathbf{B}^{b}\right]^{T} \cdot \boldsymbol{\sigma}_{n+1}^{\prime s}\left(\mathbf{u}_{n+1}^{(e)}\right) d \Omega+\int_{\Omega^{e}} b N_{, i}^{b} p_{n+1}^{(e)} d \Omega \\
& p \leftarrow i+n d f(b-1) \quad / / b=1, \text { nen }
\end{aligned}
$$

else if $(e=$ pressure element $)$ then

$$
\begin{aligned}
& r_{p, n+1}^{(i)} \leftarrow f_{n+1}^{b, e x t}-\int_{\Omega^{e}} \frac{1}{M} N^{b} \dot{p}_{n+1}^{(e)} d \Omega-\int_{\Omega^{e}}\left[\nabla N^{b}\right]^{T} \cdot \frac{\mathbf{k}}{\mu_{f}} \cdot \nabla p_{n+1}^{(e)} d \Omega-\int_{\Omega^{e}} b N^{b} \nabla \cdot \dot{\mathbf{u}}_{n+1}^{(e)} d \Omega \\
& p \leftarrow n s d+1+n d f(b-1)
\end{aligned}
$$

end if $e$

$J_{p q}^{(e)} \leftarrow \frac{r_{p, n+1}^{(i)}\left(\dot{\mathbf{x}}_{n+1}^{(i)}+h\right)-r_{p, n+1}^{(i)}\left(\dot{\mathbf{x}}_{n+1}^{(i)}\right)}{h}$

$/ /$ recall $p=1$, nen $\times n d f, q=i d o f+n d f(a-1)$

end do idof

end do $a$

$[\mathbf{J}] \leftarrow[\mathbf{J}]+A_{e}\left[J_{p q}^{(e)}\right] \quad / /$ assembly

end do $e$ 
At the core of the combined simultaneous solution procedure is the assembly and solution of a set of non-symmetric (linear) equations. However, one can entirely avoid forming the full Jacobian matrix equation by using a partitioned iterative conjugate gradient procedure (Hestnes and Stiefel, 1952) to solve for the Schur complement as detailed in Prevost (1997). Although correct, aside from the usual errors associated with spatial and temporal discretizations, the simultaneous fully coupled solution of the geomechanical and reservoir equations still places high demand on computational resources.

\section{NUMERICAL RESULTS}

Numerical simulations in 2D and 3D are presented, which demonstrate the accuracy and versatility of the proposed X-FEM faults models.

\subsection{Fluid-flow in a 2D domain}

The problem geometry, and boundary conditions used are shown in Fig. 4. The dimensions of the two-dimensional domain are $L_{X}=L_{Y}=20 \mathrm{~m}$. The material parameters are shown in Table 2. In this example, the rock permeability used is $k_{\text {rock }}=3.33 \times 10^{-14} \mathrm{~m}^{2}$, and an inflow fluid flux $q_{f}=10^{-5} \mathrm{~m}^{3} / \mathrm{s}$ is prescribed on the top boundary. No flow is assumed to take place on both lateral boundaries, and a free-flow boundary is used at the bottom where the fluid pressure $p_{f}=0$. The fault is inclined at $\alpha=45^{\circ}$ and is assigned a thickness $t=0.1 \mathrm{~m}$. Note that the thickness assigned to the fault in this example is arbitrarily large in order to be able to mesh the fault when using a standard finite element solution procedure. The problem is first solved by using a standard finite element procedure and by meshing both the domain and the fault as shown in Fig. 5 on an unstructured mesh. The mesh consists of 19,724 nodes and 39,366 triangular elements (30,140 for the domain and 9,226 for the fault). The fault permeability in the global axes is computed by performing the rotation of the fault local permeability $\mathbf{k}_{\text {local }}$ with transverse and longitudinal permeability $k_{n}$ and $k_{t}$ respectively, to the reference axes of the domain, viz. $\mathbf{k}=\mathbf{R}^{T} \cdot \mathbf{k}_{\text {local }} \cdot \mathbf{R}$ (see (25)).

The resulting pressure contours are shown in Fig. 5c for a fault with transverse and longitudinal permeability $k_{n}=10^{-2} k_{\text {rock }}, k_{t}=10^{+3} k_{\text {rock }}$, respectively. The maximum fluid pressure is computed as $5.95 \mathrm{MPa}$. 


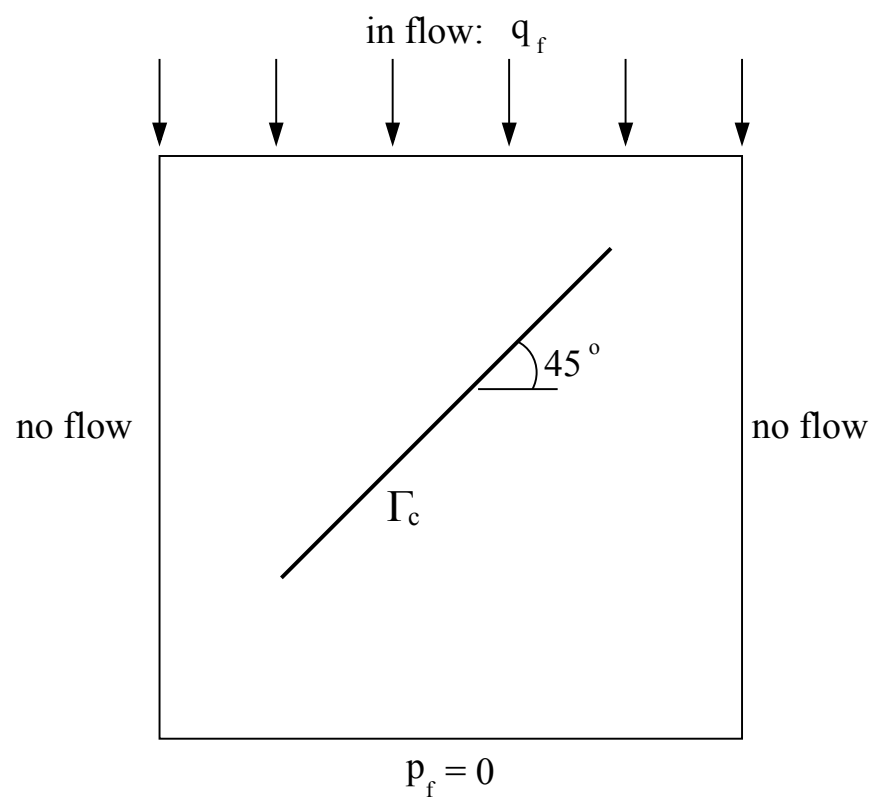

Fig. 4: Geometry and boundary conditions for fluid flow in a 2D domain.

Table 2: Materials properties.

Permeability (host rock, isotropic)

Fluid viscosity (water)

Porosity

Young's modulus

Poisson's ratio

Fluid bulk modulus

Fault friction angle

Fault cohesion

$\begin{array}{ll}k_{\text {rock }} & 3.33 \times 10^{-14} \mathrm{~m}^{2}-1.71 \times 10^{-15} \mathrm{~m}^{2} \\ \mu_{f} & 10^{-3} \mathrm{~Pa} \\ \varphi & 0.30 \\ E^{S} & 30 \mathrm{GPa} \\ v^{S} & 0.0 \\ K_{f} & 2.17 \mathrm{GPa} \\ \varphi_{u} & 34^{\circ}-40^{\circ} \\ c_{u} & 0.0\end{array}$




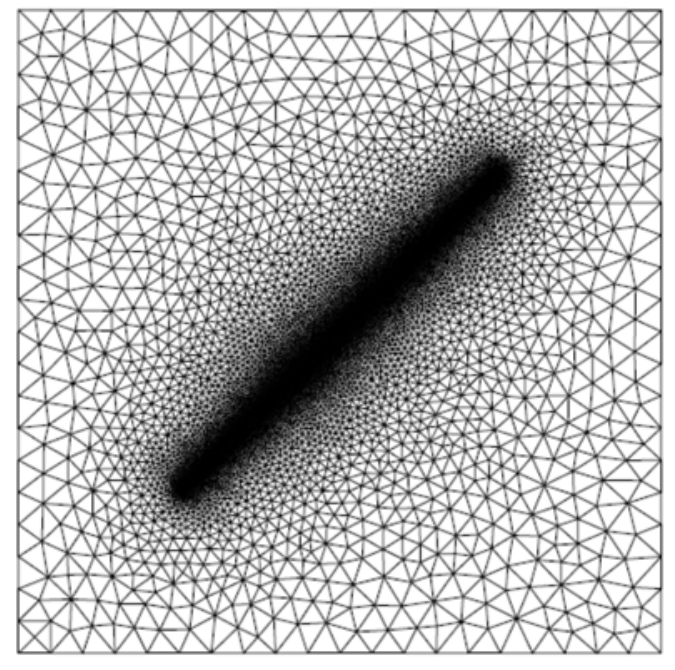

(a)

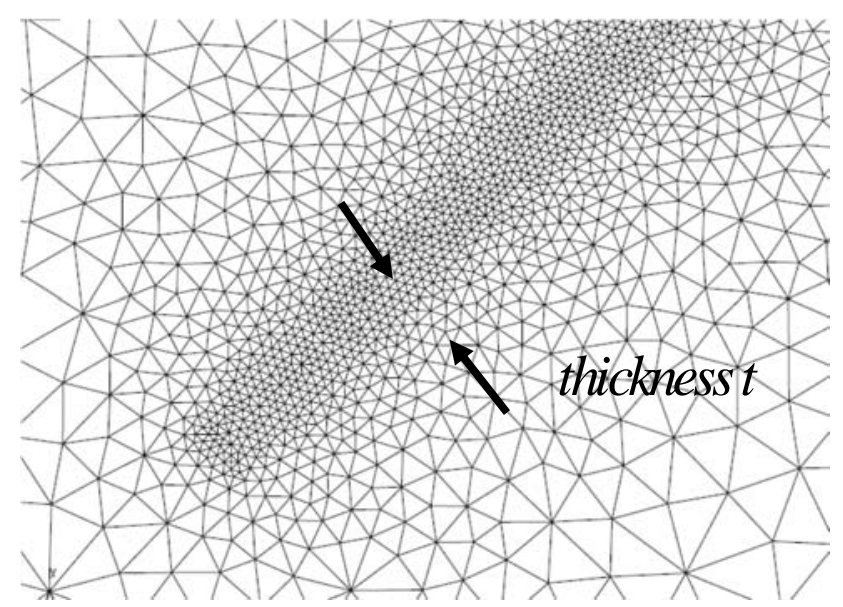

(b)

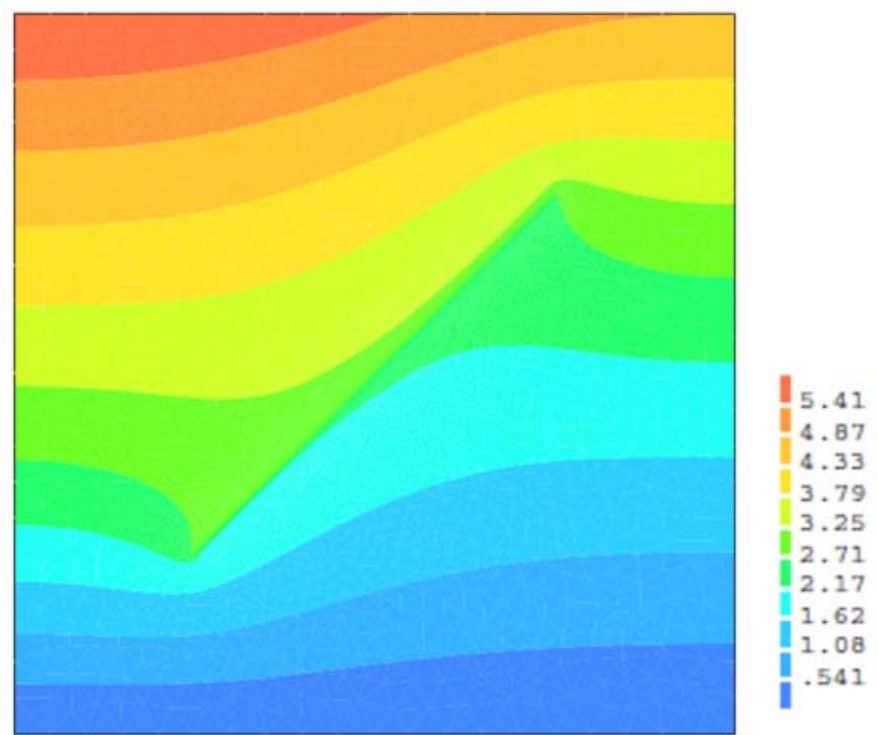

(c)

Fig. 5: Finite element solution for the fluid-flow problem. (a) Refined mesh, (b) Zoom view of the mesh at the fault-bottom where the fault thickness, $t=0.1 \mathrm{~m}$ is shown, and (c) Pressure contour. The fault permeability is: $k_{n}=10^{-2} k_{\text {rock }}$ and $k_{t}=10^{3} k_{\text {rock }}$. The maximum computed fluid pressure, $p_{f, \max }$, is $5.95 \mathrm{MPa}$.

The same problem is solved with the X-FEM pressure option (fault as a conduit) using a structured quadrilateral mesh. The results are shown in Fig. 6 for a mesh size $h=2 \mathrm{~m}$. Note that even with such a coarse mesh, the fluid pressures results are accurate when compared with Fig. 5c. Also, the computed maximum fluid pressure 5.52 MPa compares well with the one computed by meshing 
the fault $(5.95 \mathrm{MPa})$. The flow in the cross section of the fault in the FEM solution is found to be highly non-uniform, which the X-FEM procedure cannot reproduce since it lumps it into a single flow in and out of the fault channel. So the extended FE solution is not able to capture the details of the flow in the fault, and can only provide an average solution. This example demonstrates the significant simplifications with reasonable accuracy of the extended FE fault model for the fluid flow. Clearly, the fault permeability used in the example is only representative. However the extended FE procedure can easily accommodate other values as dictated by Geologist experts.

In order to assess the accuracy and stability of the X-FEM, a systematic mesh sensitivity study was performed as summarized in Fig. 7 which shows the X-FEM computed maximum fluid pressure (in $\mathrm{MPa}$ ) as a function of the nodal spacing h on a log-scale (in $\mathrm{m}$ ), and demonstrates that even with coarse meshes the solution can be captured with sound accuracy, and demonstrate the stability of the procedure. Note that the fault thickness used is $0.1 \mathrm{~m}$, and therefore the nodal spacing must be greater (or equal) than $0.1 \mathrm{~m}$. In Fig. 7, the X-FEM CPU times are also reported by normalizing them with respect to the FEM times. The 2D FEM mesh has 19,203 dofs and takes $5 \mathrm{sec}$ to compute. The mesh and solution parameters for the extended FE analyses are: $\mathrm{h}=0.1 \mathrm{~m}$ (40,906 dofs), $0.2 \mathrm{~m}$ (10,456 dofs), $0.4 \mathrm{~m}$ (2734 dofs), $1.0 \mathrm{~m}$ (496 dofs), $2.0 \mathrm{~m}$ (154 dofs), and 4.0 $\mathrm{m}$ (30 dofs). Note that $h=0.1 \mathrm{~m}$ is not really meaningful since the fault thickness must be smaller than the mesh size (see Section 4.2), and has more equations than the FEM mesh (and thus takes longer to compute). However, it is clear that the X-FEM provides marked savings over the FEM (Fig. 5a and 5b).
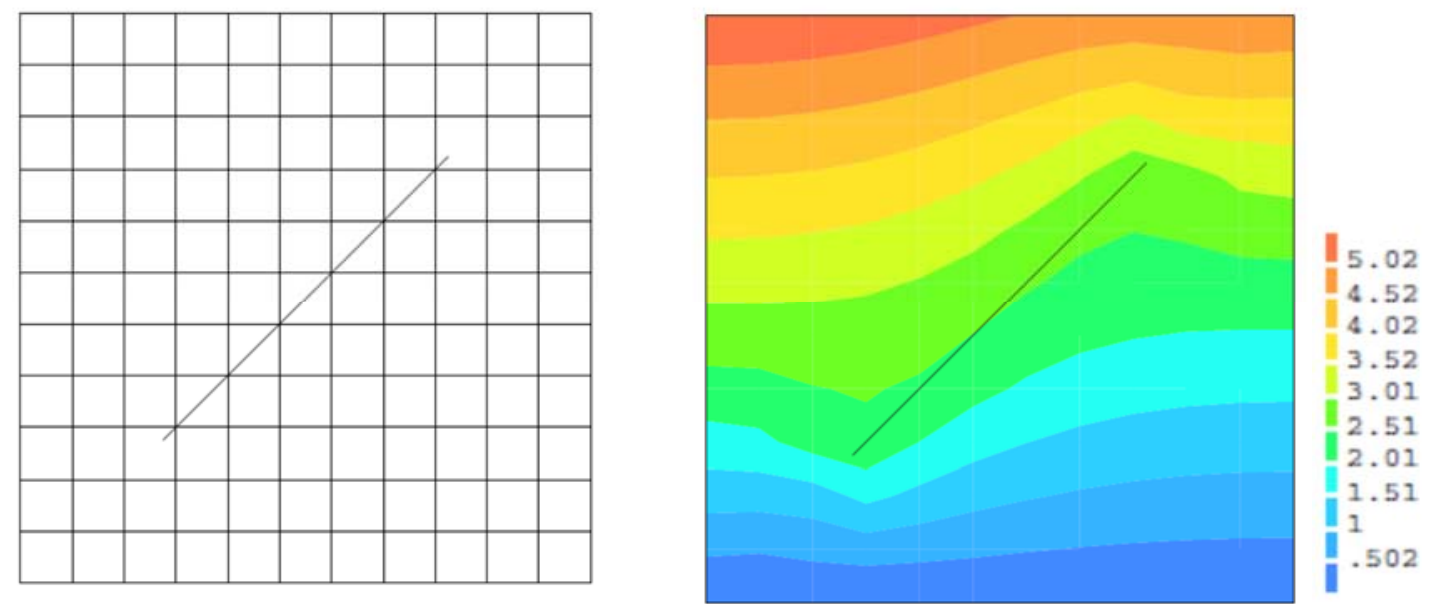

Fig. 6: Fluid flow problem solved using the X-FEM. Mesh (left) and pressure contour (right). Mesh size $h=20 / 11 \approx 2.0 \mathrm{~m}$. The fault permeability is $k_{n}=10^{-2} k_{\text {rock }}$ and $k_{t}=10^{3} k_{\text {rock }}$. The fault thickness, $t$, is $0.1 \mathrm{~m}$ and the maximum pressure, $p_{f, \max }$, is $5.52 \mathrm{MPa}$. 


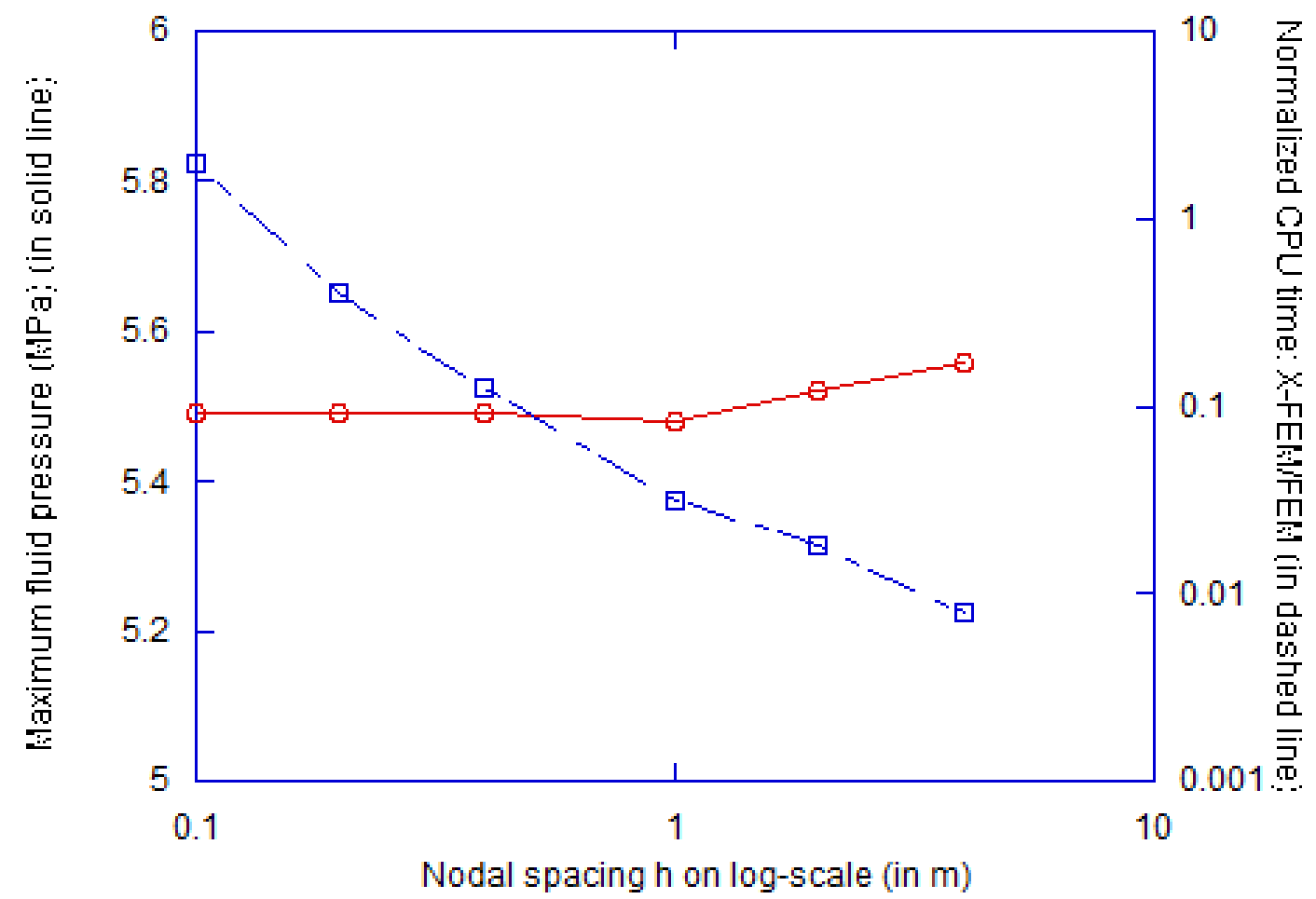

Fig. 7: Mesh sensitivity results and normalized CPU times (X-FEM/FEM)

\subsection{Fluid flow in a 3D domain}

In this example, a three-dimensional cube $40 \mathrm{~m} \times 40 \mathrm{~m} \times 40 \mathrm{~m}$ is cut by two faults at angles $\alpha_{1}=-35^{\circ}$ and $\alpha_{2}=55^{\circ}$, respectively. The material parameters are shown in Table 2 . In this example the rock permeability used is $k_{\text {rock }}=3.33 \times 10^{-14} \mathrm{~m}^{2}$, and a uniform inflow fluid flux $q_{f}=10^{-6} \mathrm{~m}^{3} / \mathrm{s}$ is prescribed on the top boundary. No flow is assumed to take place on all lateral boundaries, and a free-flow boundary is used at the bottom where the fluid pressure $p_{f}=0$. The domain and boundary conditions are shown schematically in Fig. 8. The fault acting as a flow conduit is assigned a thickness $t=h \times 10^{-2}=0.02 \mathrm{~m}$. Fault 1 is assumed to be a flow conduit, and its transverse and longitudinal permeabilities are chosen as $k_{n}=10^{-3} k_{\text {rock }}$ and $k_{t}=10^{3} k_{\text {rock }}$, respectively. Fault 2 is assumed to be a sealing fault and is assigned a permeability $k_{n}=k_{t}=10^{-4} k_{\text {rock }}$. The unstructured mesh is shown in Fig. 9a and contains 3,223 nodes and 16,281 tetrahedral elements. The computed fluid pressures are shown in Fig. $9 \mathrm{~b}$ (maximum fluid pressure $=1.51 \mathrm{MPa}$ ), and the resulting Darcy fluid velocities are shown in Fig. 9c. Note that most of the flow is taking place along the fault plane of fault 1 due to its high longitudinal permeability (and low transverse permeability). 


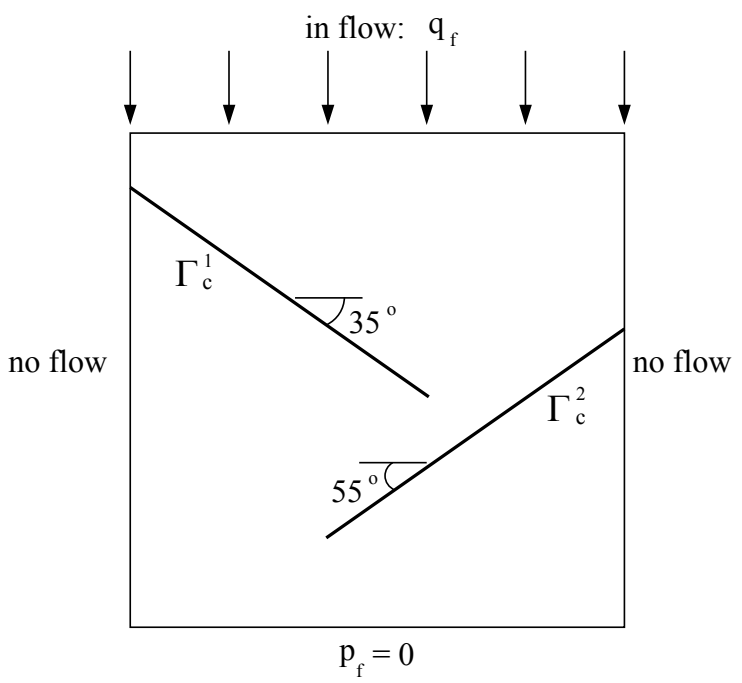

Fig. 8: Geometry and boundary conditions for fluid flow in a 3D domain.

The example presented in this Section illustrates the capability of modeling two faults in three dimensions. Modeling many (tens or even hundreds) faults is relatively straightforward since the faults are never meshed, as they are just placed within the reservoir mesh by simply identifying their plane(s). This is illustrated by the example shown in Fig. 10 in which four faults are now present. Faults 1-3 are flow conduit as in Fig. 9, and fault 4 is assumed to be a sealing fault. The $\mathrm{X}-\mathrm{FEM}$ mesh is shown in Fig. 10a. The computed fluid pressures are shown in Fig. 10b and the resulting Darcy fluid velocities are shown in Fig. 10c. As noted in the previous example, most of the flow occurs along the fault planes of the faults labeled as 1, 2 and 3 that are modeled as flow conduits.

\subsection{Stress in a $2 D$ and $3 D$ domain}

In this example, a three-dimensional cube $40 \mathrm{~m} \times 40 \mathrm{~m} \times 40 \mathrm{~m}$ is cut by a fault at an angle $\alpha=35^{\circ}$. A uniform total vertical surface traction is applied on the top surface. The geomechanical material parameters are given in Table 2. The unstructured mesh is shown in Fig. 11a and contains 3,223 nodes and 16,281 tetrahedral elements. The resulting displacement vectors for a fault friction angle $\varphi_{u}=40^{\circ}$ (no slip) are shown in Fig 11b. The resulting displacement vectors for a fault friction angle $\varphi_{u}=34^{0}$ (slip) are shown in Fig 11c. The same problem is solved as a 2D problem (plane strain). The structured mesh is shown in Fig. 12a, and the resulting displacement vectors for a fault friction angle $\varphi_{u}=40^{\circ}$ (no slip) and for a fault friction angle $\varphi_{u}=34^{0}$ (slip) are shown in Fig. 12 b and Fig. 12 c, respectively. Note that we used a Poisson's ratio $v^{S}=0$ (Table 2) to ensure consistency between the $2 \mathrm{D}$ and $3 \mathrm{D}$ results, without having to resort to boundary conditions on the lateral faces of the $3 \mathrm{D}$ mesh. This example demonstrates the accuracy of the stress fault model. Note that no numerical stability issues on the contact tractions on the fault surface such as alluded to in Annavarapu et al. (2015) are encountered in our procedure. This may be due to the fact that we use a penalty formulation (see Section 4.1), and do not attempt to analytically derive the tangent operators arising from the Coulomb stick/slip logic on the interface. No matrix is 
defined explicitly because we use a numerical finite differencing of the residuals to compute numerically those coupled matrices as discussed in Section 5.3. Our procedure is entirely general and bypasses analytically defined matrices, which in general are difficult (or impossible to define) especially in our case and have the advantage of being consistent with time stepping, mesh size and nonlinear algorithmic details.

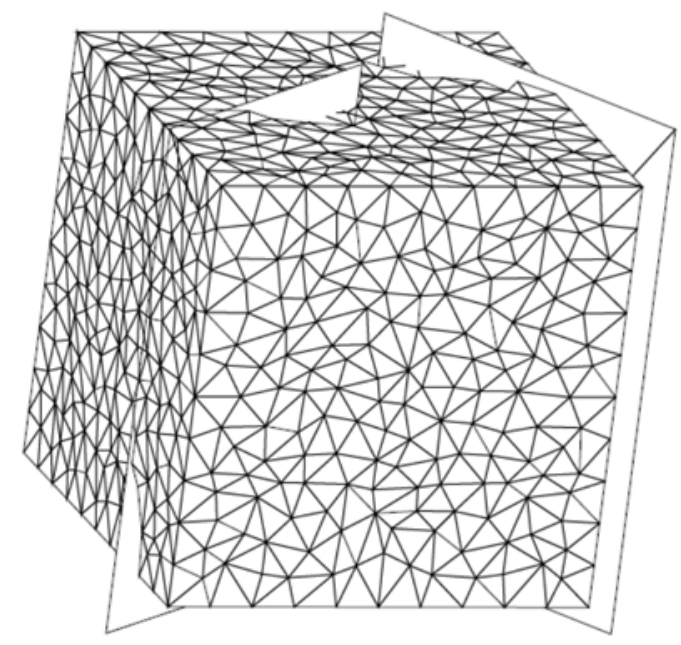

(a)

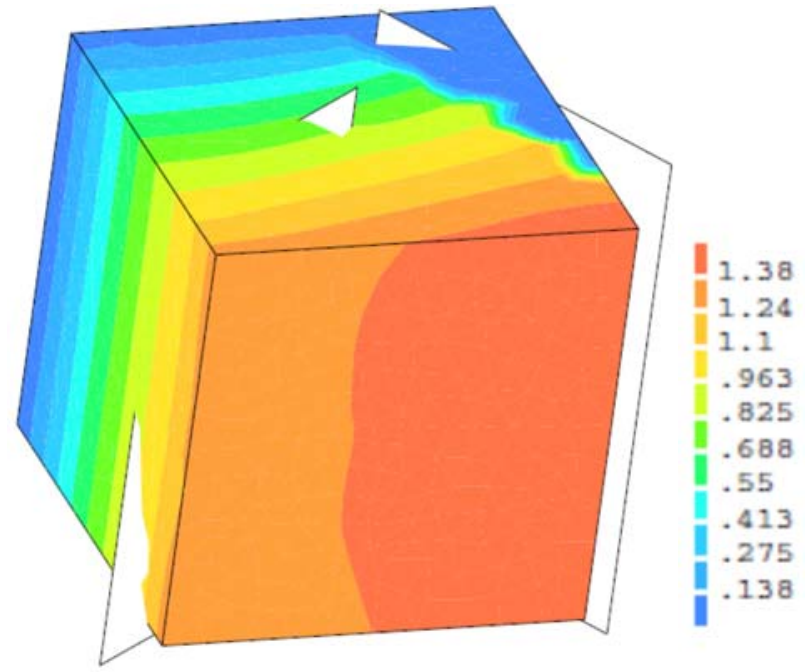

(b)

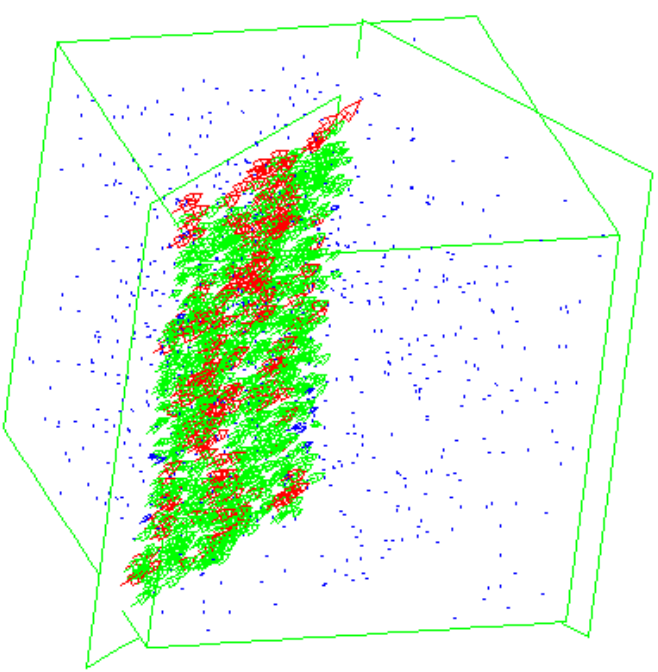

(c)

Fig. 9: Extended FE solution for the fluid flow in a 3D domain. (a) Unstructured mesh (two faults are present); (b) Fluid pressure distribution; maximum fluid pressure is $1.51 \mathrm{MPa}$; (c) Darcy velocities; maximum velocity is $5.2 \times 10^{-4} \mathrm{~m} / \mathrm{s}$. 


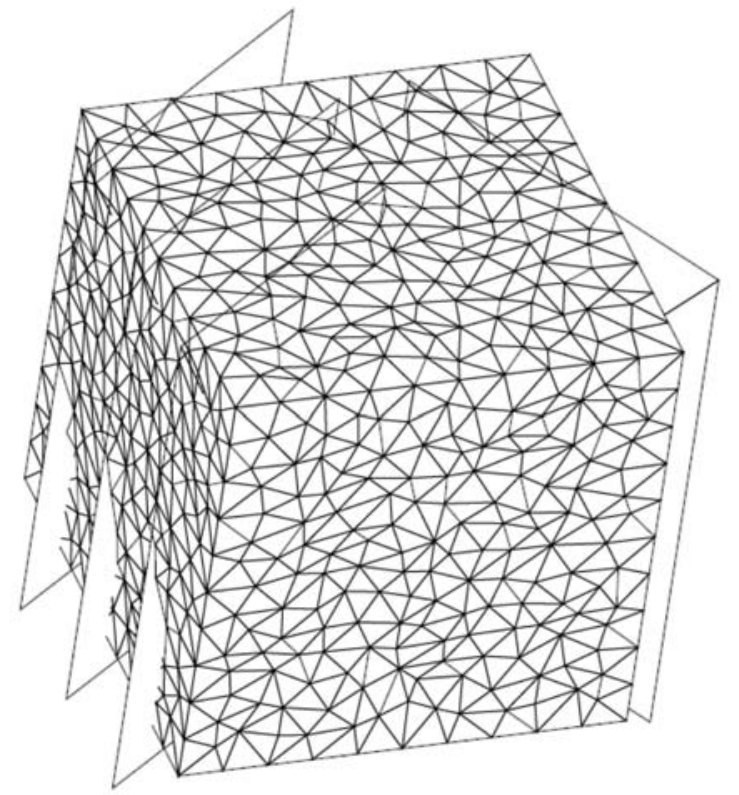

(a)

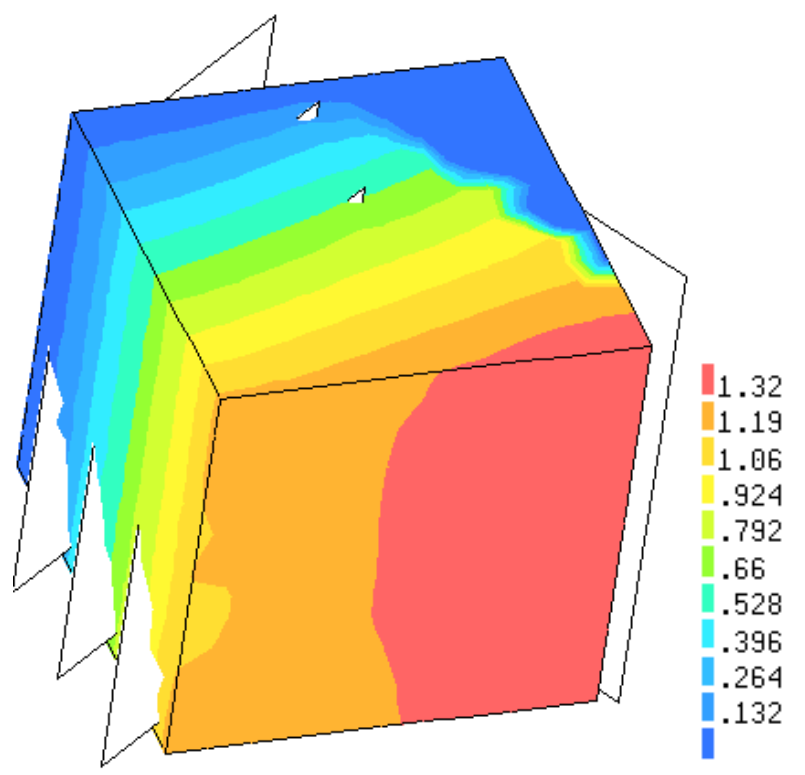

(b)

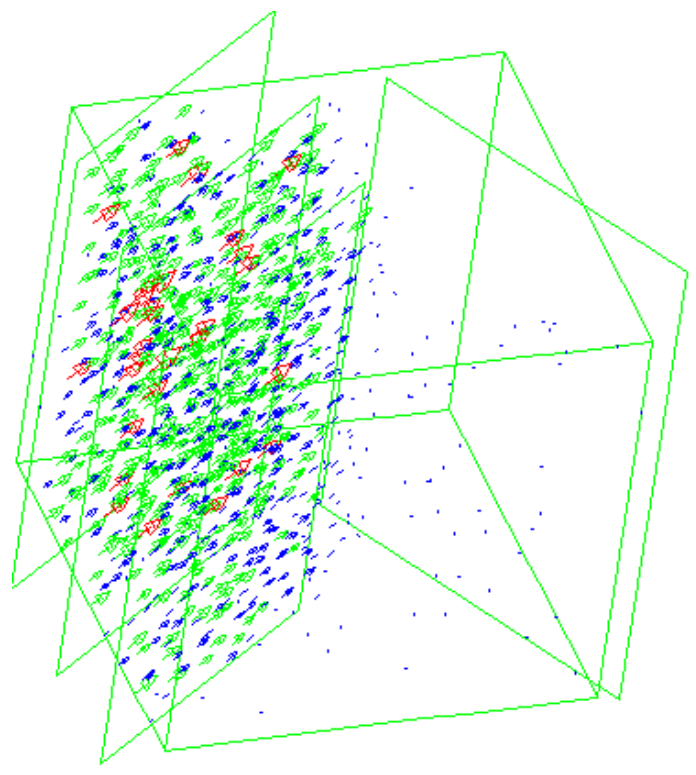

(c)

Fig. 10: Extended FE solution for the fluid flow in a 3D domain. (a) Unstructured mesh (four faults are present); (b) Fluid pressure distribution; maximum fluid pressure is $1.45 \mathrm{MPa}$; (c) Darcy velocities; maximum velocity is $4 \times 10^{-8} \mathrm{~m} / \mathrm{s}$. 


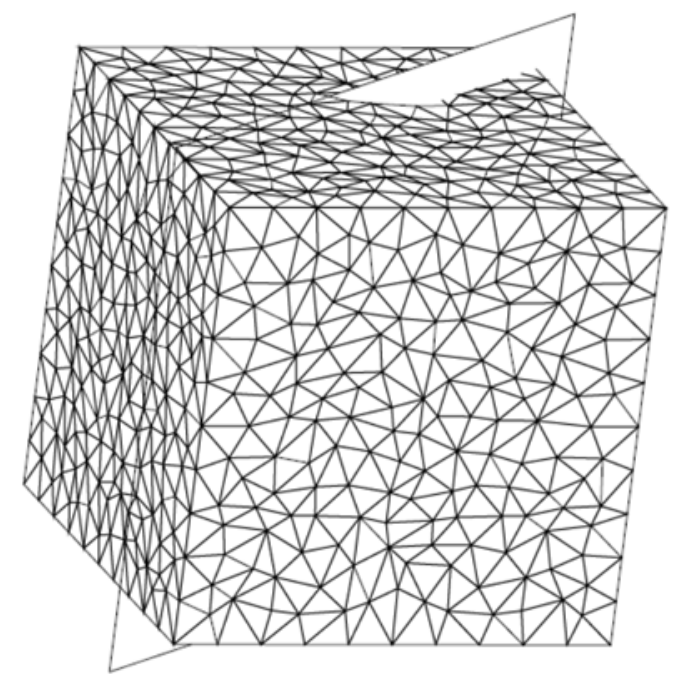

(a)

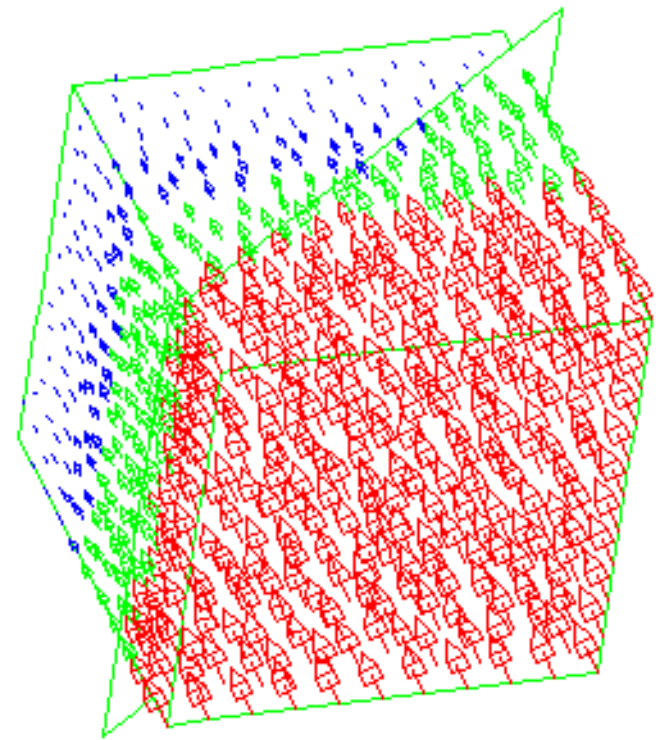

(b)

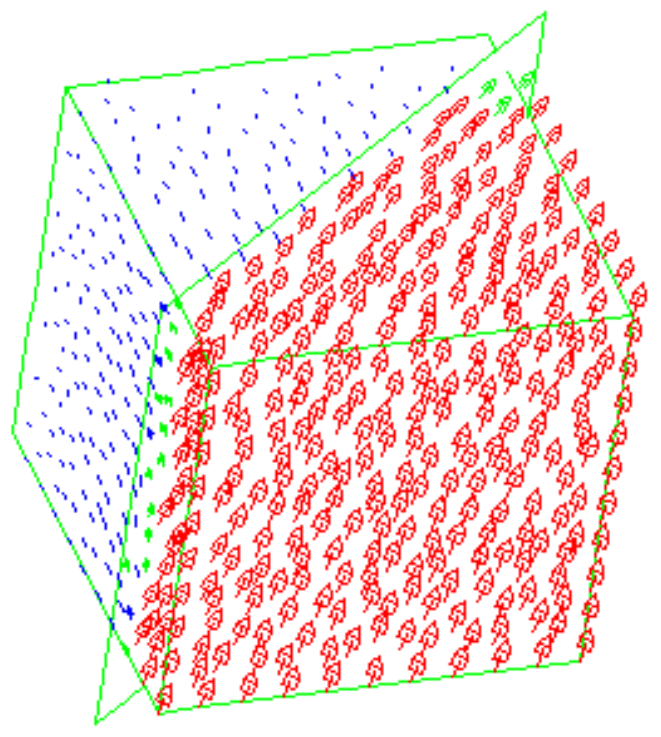

(c)

Fig. 11: Extended FE solution for 3D stress fault model. (a) Unstructured mesh, with fault intersecting at an angle $\alpha=35^{\circ}$; (b) Displacement vectors show no slip along the fault for friction angle $\varphi_{u}=40^{\circ}$; (c) Displacement vectors show slip along the fault for friction angle

$$
\varphi_{u}=34^{0} .
$$




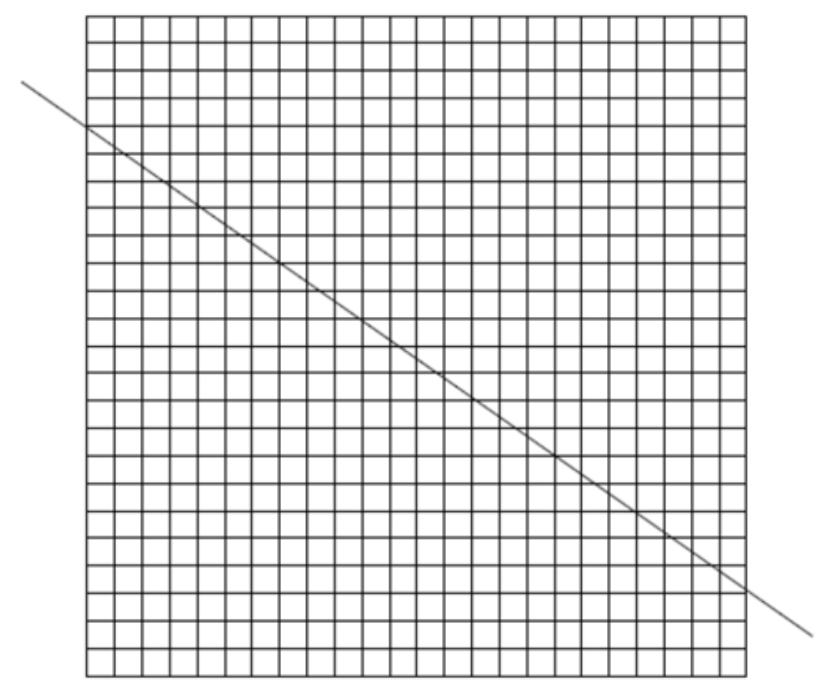

(a)

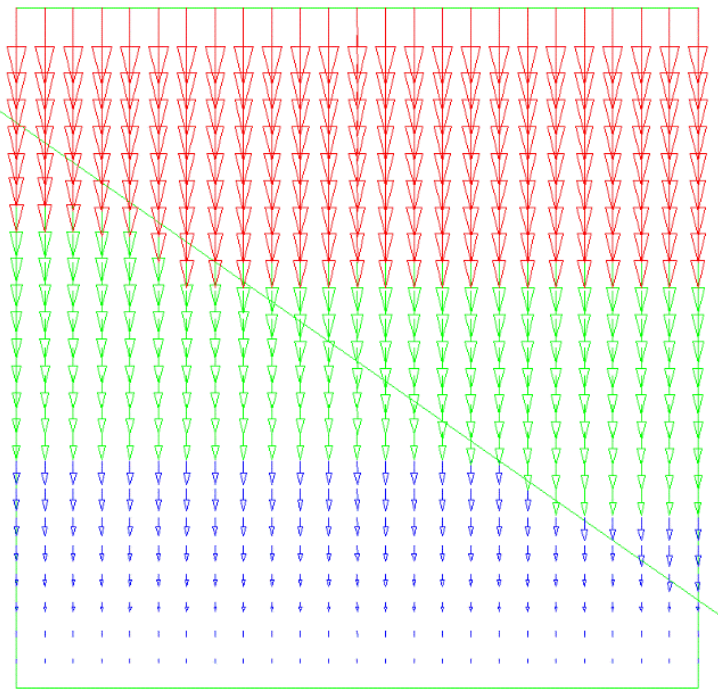

(b)

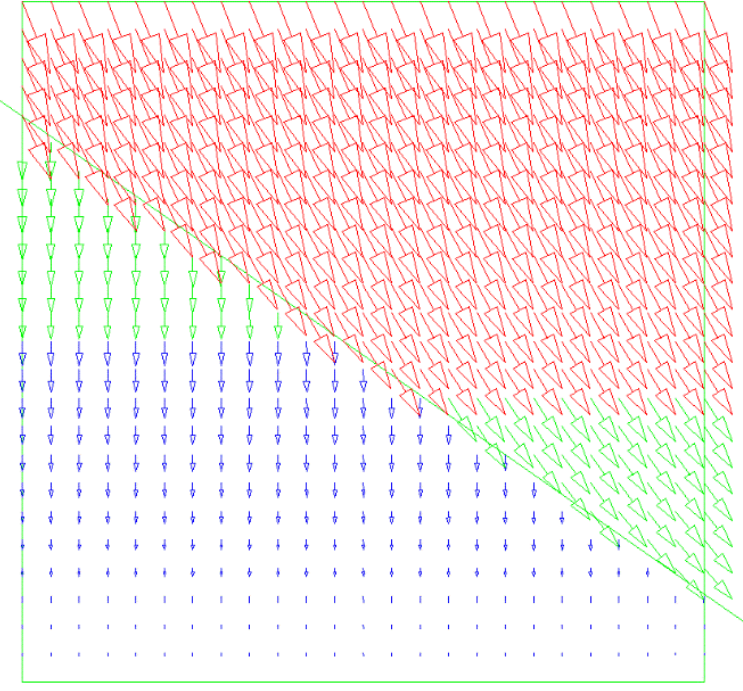

(c)

Fig. 12: Extended FE solution for 2D stress fault model. (a) Structured mesh, with fault intersecting at an angle $\alpha=35^{\circ}$; (b) Displacement vectors show no slip along the fault for friction angle $\varphi_{u}=40^{\circ}$; (c) Displacement vectors show slip along the fault for friction angle

$$
\varphi_{u}=34^{0} .
$$




\subsection{Fully-coupled reservoir-geomechanics}

The problem geometry and boundary conditions are shown in Fig. 13. The dimensions of the twodimensional domain are $L_{X}=L_{Y}=10 \mathrm{~m}$. The material parameters are shown in Table 2. In this example, the rock permeability is: $k_{\text {rock }}=1.71 \times 10^{-15} \mathrm{~m}^{2}$, and a sealing fault is used with permeability $k_{n}=k_{t}=10^{-5} k_{\text {rock }}$. The fault is inclined at an angle $\alpha=35^{\circ}$, and the fault friction angle is assumed to be $\varphi_{u}=40^{\circ}$. A time-dependent uniform total surface traction with initial value $h_{0}=5 \mathrm{MPa}$ is applied on the top boundary and a fixed fluid pressure $p_{f 0}=1 \mathrm{MPa}$ is applied on the top boundary. No flow is assumed to take place on both lateral and bottom boundaries. The time scale of this problem is controlled by the diffusion parameter $c_{f}$ (see (6)) and for this case $c_{f}=10^{-2} \mathrm{~m}^{2} / \mathrm{s}$. Depending on the loading rate, the response is expected to exhibit very different features. Two time scales are investigated: rapid (i.e., undrained) and slow (i.e., drained) loading, respectively, as shown in Fig. 14.

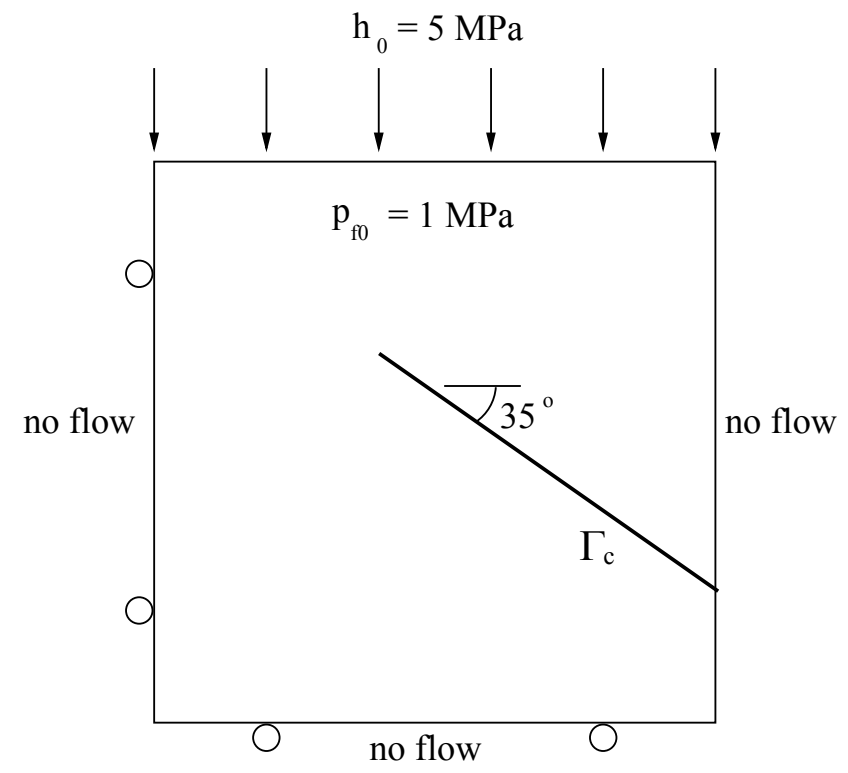

Fig. 13: Geometry and boundary conditions for the fully-coupled reservoir geomechanics problem. 


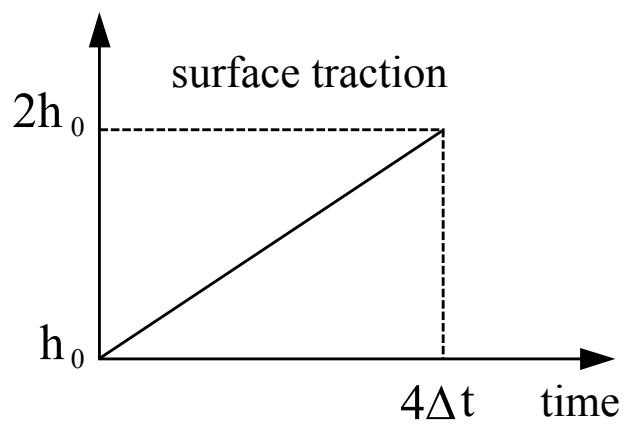

Fig. 14: Applied total surface traction time history (fast vs slow loading). For the fast loading case (undrained), $\Delta t=10^{-1} H^{2} / c_{f}$, and for the slow loading case (drained), $\Delta t=10^{2} H^{2} / c_{f}$.

Numerical results are shown in Figs 15 and 16 for both slow and rapid rates of loading. Note that as a result of the fast loading application, significant excess pore-water pressures are built on the fault (Fig. 15b) and reactivate the fault (Fig. 16b). On the other hand, no significant pore-water pressures can build on the fault during the slow loading (Fig. 15a) and the fault remains stable (Fig. 16a). This example demonstrates the influence of rate of loading on activation of faults. It is also interesting to note that for a rock permeability of the order $10^{-15} \mathrm{~m}^{2}=10^{-3}$ Darcy $=1$ milliDarcy , which may be applicable to sandstones $\left(c_{f}=10^{-2} \mathrm{~m}^{2} / \mathrm{s}\right)$, the fast loading rate time scale is of the order of 0.3 hours, whereas the slow loading rate is of the order of 300 hours. For shales with permeability of the order $10^{-21} \mathrm{~m}^{2}=10^{-9}$ Darcy $=1$ nanoDarcy $\left(c_{f}=10^{-8} \mathrm{~m}^{2} / \mathrm{sec}\right)$ the fast loading rate time scale is of the order of 400 months. It may be concluded that the fast loading rate (i.e., undrained) is therefore the norm for engineered processes in the subsurface. This example reveals the influence of the rate of loading on the activation of faults.

\section{CONCLUSIONS}

In this paper, we introduced faults in a finite element mesh without requiring them to conform to the mesh, by using the extended finite element method (X-FEM), in which faults are represented by enriching the displacement and/or pressure approximations through the framework of partitionof-unity (Melenk and Babuška, 1996). The proposed approach is a marked departure from the FEM for the modeling of faults. Significant meshing difficulties arise when faults (multiple) are modeled using the FEM, where the mesh has to conform to the geometry of the fault(s) and hence a structured mesh cannot be used. Unstructured mesh generation (triangles in $2 \mathrm{D}$ and tetrahedra in 3D) is needed for the FEM (see Figures 5a and 5b). On using the X-FEM, a structured mesh suffices and the faults can arbitrarily cut the elements in the mesh. This advantage of the X-FEM has been leveraged for many applications in fracture mechanics, and in this paper we have shown that it is equally advantageous for modeling faults. 


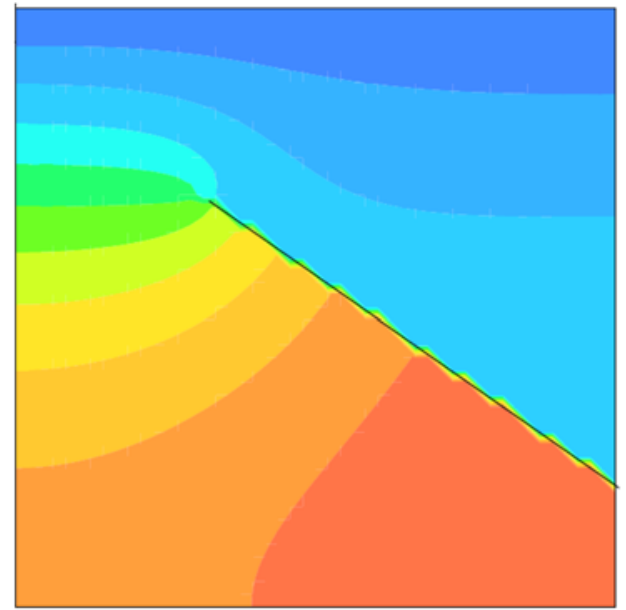

(a)

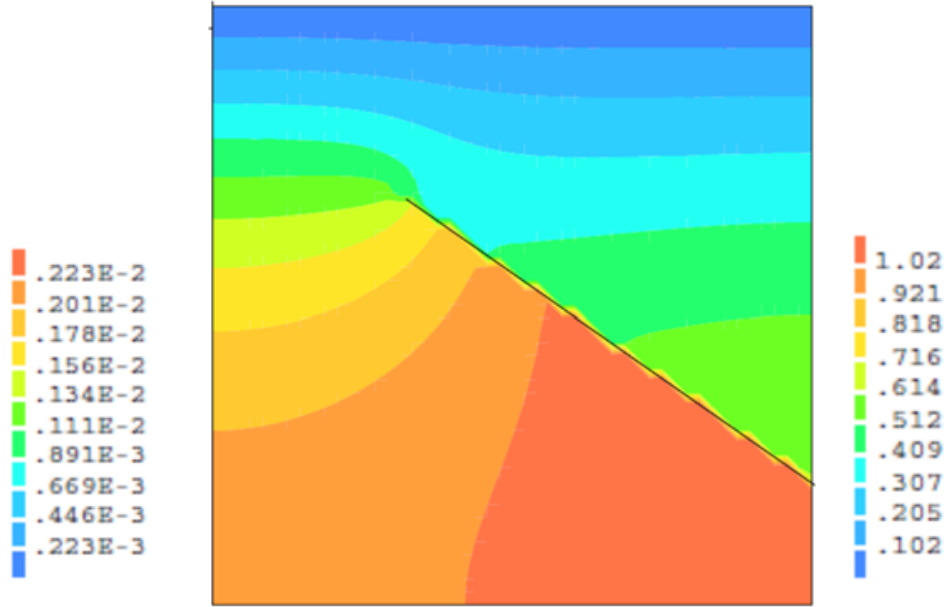

(b)

Fig. 15: Extended FE solution for the reservoir-geomechanics problem with excess fluid pressure.

(a) Drained case, $\Delta p_{f}=2.4 \times 10^{-3} \mathrm{MPa}$; (b) Undrained case, $\Delta p_{f}=1.13 \mathrm{MPa}$.

In the X-FEM, the nodes whose basis function support intersects the fault are enriched. For the geomechanics, the fault is treated as an internal displacement discontinuity that allows slipping to occur using a Mohr-Coulomb type criterion. For the reservoir, the fault is either an internal fluid flow conduit that allows fluid flow in the fault, as well as to enter/leave the fault or a barrier to flow (sealing fault). In the X-FEM, the faults are represented by enriching the displacement approximation with a discontinuous (generalized Heaviside) function. For internal fluid flow conduits, the approximation for the fluid pressure uses continuous functions that admit a discontinuity in their normal derivative across the fault. For sealing/impermeable faults a discontinuous (generalized Heaviside) function is used to model pressure discontinuities across the fault. The procedure has been implemented in both 2D and 3D for both structured and unstructured meshes. Examples that demonstrate the versatility and accuracy of the procedure(s) were presented, and the influence of rate of loading on the activation of faults was also assessed. Modeling many (tens or even hundreds) faults is relatively straightforward since the faults are never meshed and hence they can be placed within the reservoir mesh by simply identifying their plane(s). However, we point out that there does exist a limitation in our present implementation, namely, we currently cannot accommodate nonplanar faults in $3 \mathrm{D}$, which presents significant additional challenges. This is the subject of ongoing research and we hope to overcome this limitation in a future communication. 


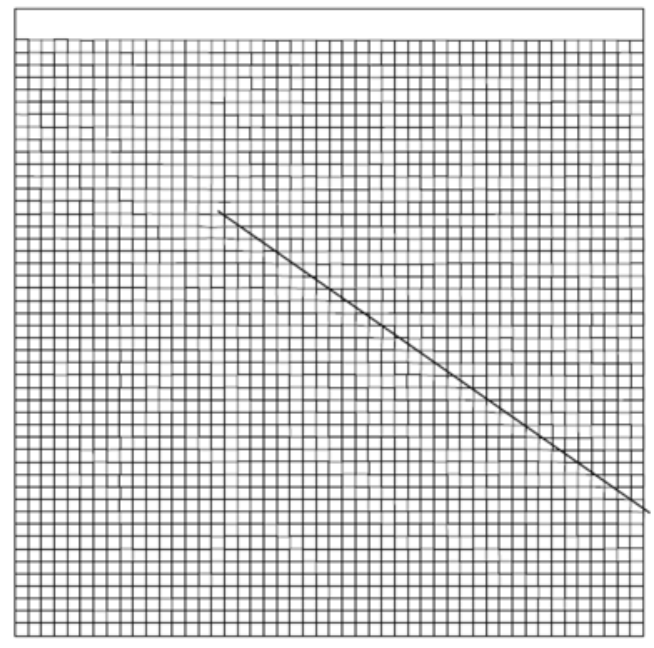

(a)

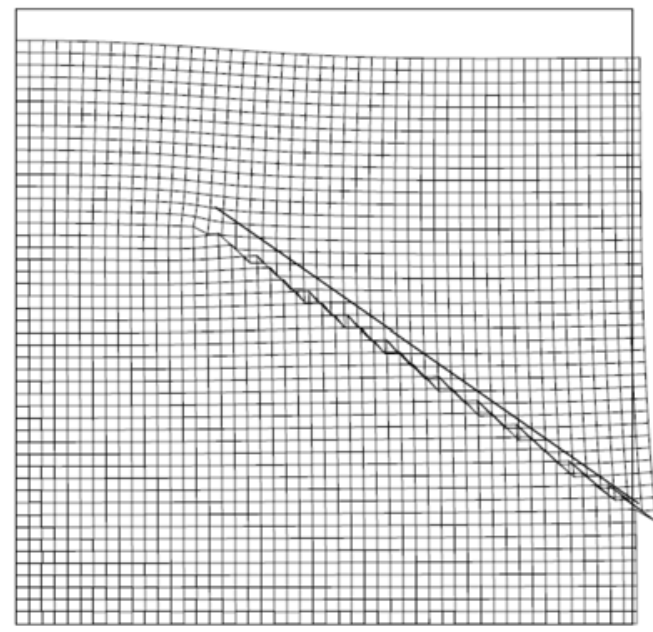

(b)

Fig. 16: Slip as a function of the loading rate. (a) No slip (magnification factor $=300$ ) for the drained case with $\Delta t=10^{2} H^{2} / c_{f}$, and (b) Slip (magnification factor $=300$ ) for the undrained case with $\Delta t=10^{-1} H^{2} / c_{f}$.

\section{ACKNOWLEDGEMENTS}

Funding for this research was partially provided by Princeton University DYNAFLOW research fund. We also thank Linda Everett and Eric Chin for their assistance with the typesetting of the manuscript. 


\section{REFERENCES}

Annavarapu, C., Settgast, R.R., Johnson, S.M., Fu, P., Herbold, E.B., 2015. A weighted Nitsche stabilized method for small-sliding contact on frictional surfaces. Comput. Methods Appl. Mech. Eng. 283, 763-781. DOI: http://dx.doi.org/10.1016/j.cma.2014.09.030.

Armero, F., Callari, C., 1999. An analysis of strong discontinuities in a saturated poro-plastic solid. Int. J. Numer. Meth. Eng. 46, 1673-1698. DOI: 10.1002/(SICI)10970207(19991210)46:10<1673::AID-NME719>3.0.CO;2-S.

Barton, N., 2013. Shear strength criteria for rock, rock joints, rockfill and rock masses: Problems and some solutions. J. Rock Mech. Geotech. Engng. 5, 249-261. DOI: 10.1016/j.jrmge.2013.05.008.

Biot, M.A., 1941. General theory of three-dimensional consolidation. J. Appl. Phys. 12, 155-164.

Biot, M.A., 1955. Theory of elasticity and consolidation for a porous anisotropic solid. J. Appl. Phys. 26, 182-185.

Cappa, F., Rutqvist, J., 2011. Modeling of coupled deformation and permeability evolution during fault reactivation induced by deep underground injection of CO2. Int. J. Greenh. Gas Con. 5, 336-346. DOI: 10.1016/j.ijggc.2010.08.005.

Coates, R.T., Schoenberg, M., 1995. Finite-difference modeling of faults and fractures. Geophysics 60, 1514-1526. DOI: 10.1190/1.1443884.

Coussy, O., 2004. Poromechanics. Wiley, Chichester, England.

de Borst, R., Réthoré, J., Abellan, M.-A., 2006. A numerical approach for arbitrary cracks in a fluid-saturated medium. Arch. Appl. Mech. 75, 595-606. DOI: 10.1007/s00419-006-0023y.

Evans, J.P., Forster, C.B., Goddard, J.V., 1997. Permeability of fault-related rocks, and implications for hydraulic structure of fault zones. J. Struct. Geol. 19, 1393-1404. DOI: 10.1016/S0191-8141(97)00057-6.

Faulkner, D.R., Lewis, A.C., Rutter, E.H., 2003. On the internal structure and mechanics of large strike-slip fault zones: field observations of the Carboneras fault in southeastern Spain. Tectonophysics 367, 235-251. DOI: 10.1016/S0040-1951(03)00134-3.

Fumagalli, A., Scotti, A., 2014. An efficient XFEM approximation of Darcy flows in arbitrarily fractured porous media. Oil Gas Sci. Technol. - Rev. IFP Energies Nouvelles 69, 555-564.

Gupta, P., Duarte, C.A., 2014. Simulation of non-planar three-dimensional hydraulic fracture propagation. Int. J. Numer. Anal. Met. 38, 1397-1430. DOI: 10.1002/nag.2305.

Hestnes, M.R., Stiefel, E., 1952. Method of conjugate gradients for solving linear systems. J. Res. Nat. Bur. Stand. 49, 409-436.

Hughes, T.J.R., 1987. The Finite Element Method. Prentice Hall.

Jaeger, J.C., Cook, N.G.W., 1969. Fundamentals of Rock Mechanics. Chapman and Hall, London.

Jha, B., Juanes, R., 2014. Coupled multiphase flow and poromechanics: A computational model of pore pressure effects on fault slip and earthquake triggering. Water Resour. Res. 50, 3776-3808. DOI: 10.1002/2013WR015175.

Lamb, A.R., Gorman, G.J., Elsworth, D., 2013. A fracture mapping and extended finite element scheme for coupled deformation and fluid flow in fractured porous media. Int. J. Numer. Anal. Met. 37, 2916-2936. DOI: 10.1002/nag.2168.

Melenk, J.M., Babuška, I., 1996. The partition of unity finite element method: Basic theory and applications. Computer Methods in Applied Mechanics and Engineering 139, 289-314. DOI: $10.1016 / \mathrm{S} 0045-7825(96) 01087-0$. 
Mizoguchi, K., Hirose, T., Shimamoto, T., Fukuyama, E., 2008. Internal structure and permeability of the Nojima fault, southwest Japan. J. Struct. Geol. 30, 513-524. DOI: 10.1016/j.jsg.2007.12.002.

Moës, N., Cloirec, M., Cartraud, P., Remacle, J.F., 2003. A computational approach to handle complex microstructure geometries. Computer Methods in Applied Mechanics and Engineering 192, 3163-3177. DOI: 10.1016/S0045-7825(03)00346-3.

Moës, N., Dolbow, J., Belytschko, T., 1999. A finite element method for crack growth without remeshing. Int. J. Numer. Meth. Eng. 46, 131-150. DOI: 10.1002/(SICI)10970207(19990910)46:1<131::AID-NME726>3.0.CO;2-J.

National Research Council, 2012. Induced Seismicity Potential in Energy Technologies. National Academies Press, Washington, DC.

Preisig, M., Prevost, J.H., 2011a. Coupled multi-phase thermo-poromechanical effects. Case study: CO2 injection at In Salah, Algeria. Int. J. Greenh. Gas Con. 5, 1055-1064. DOI: 10.1016/j.ijggc.2010.12.006.

Preisig, M., Prevost, J.H., 2011b. Stabilization procedures in coupled poromechanics problems: A critical assessment. Int. J. Numer. Anal. Met. 35, 1207-1225. DOI: 10.1002/nag.951.

Preisig, M., Prevost, J.H., 2012 Fully coupled simulation of fluid injection into geomaterials with focus on nonlinear near-well behavior. Int. J. Numer. Anal. Met. 36, 1023-1040. DOI: 10.1002/nag.1039.

Prevost, J.H., 1981. DYNAFLOW: a nonlinear transient finite element analysis program (last update 2013). Dept. Civil and Environmental Engineering, Princeton University, Princeton, NJ.

Prevost, J.H., 1997. Partitioned solution procedure for simultaneous integration of coupled-field problems. Commun. Numer. Meth. En. 13, 239-247. DOI: 10.1002/(SICI)10990887(199704)13:4<239::AID-CNM51>3.0.CO;2-2.

Prevost, J.H., 2013. One-way versus two-way coupling in reservoir-geomechanical models, in: Hellmich, C., Pichler, B., Adam, D. (Eds.), Poromechanics V: Proceedings of the Fifth Biot Conference on Poromechanics, Vienna, Austria, July 10-12, 2013. American Society of Civil Engineers, pp. 517-526.

Prevost, J.H., 2014. Two-way coupling in reservoir-geomechanical models: vertex-centered Galerkin geomechanical model cell-centered and vertex-centered finite volume reservoir models. Int. J. Numer. Meth. Eng. DOI: 10.1002/nme.4657.

Réthoré, J., Borst, R.d., Abellan, M.-A., 2007. A two-scale approach for fluid flow in fractured porous media. Int. J. Numer. Meth. Eng. 71, 780-800. DOI: 10.1002/nme.1962.

Rinaldi, A.P., Rutqvist, J., Cappa, F., 2014. Geomechanical effects on CO2 leakage through fault zones during large-scale underground injection. Int. J. Greenh. Gas Con. 20, 117-131. DOI: 10.1016/j.ijggc.2013.11.001.

Rubin, A.M., 2015. Personal communication, Princeton University.

Secchi, S., Schrefler, B.A., 2012. A method for 3-D hydraulic fracturing simulation. Int J Fract 178, 245-258. DOI: 10.1007/s10704-012-9742-y.

Sukumar, N., Chopp, D.L., Moës, N., Belytschko, T., 2001. Modeling holes and inclusions by level sets in the extended finite-element method. Computer Methods in Applied Mechanics and Engineering 190, 6183-6200. DOI: 10.1016/S0045-7825(01)00215-8.

Sukumar, N., Moës, N., Moran, B., Belytschko, T., 2000. Extended finite element method for three-dimensional crack modelling. Int. J. Numer. Meth. Eng. 48, 1549-1570. DOI: 10.1002/1097-0207(20000820)48:11<1549::AID-NME955>3.0.CO;2-A. 
Sukumar, N., Prevost, J.H., 2003. Modeling quasi-static crack growth with the extended finite element method Part I: Computer implementation. Int. J. Solids Struct. 40, 7513-7537. DOI: 10.1016/j.ijsolstr.2003.08.002.

Talebian, M., Al-Khoury, R., Sluys, L.J., 2013. A computational model for coupled multiphysics processes of $\mathrm{CO} 2$ sequestration in fractured porous media. Advances in Water Resources 59, 238-255. DOI: 10.1016/j.advwatres.2013.06.012.

Terzaghi, K., 1923. Die Berechnung der Durchassigkeitsziffer des Tones aus dem Verlauf der hydraodynamishen Spannungsercheinungen. Sitzungsber. Akad. Wiss. Wien Math.Naturwiss. 132, 125-138.

Wibberley, C.A.J., Shimamoto, T., 2003. Internal structure and permeability of major strike-slip fault zones: the Median Tectonic Line in Mie Prefecture, Southwest Japan. J. Struct. Geol. 25, 59-78. DOI: 10.1016/S0191-8141(02)00014-7. 